\title{
Constitutive modeling of cyclic deformation of metals under strain controlled axial extension and cyclic torsion
}

This paper is dedicated to the memory of Franz Ziegler

Received: 18 January 2017 / Revised: 23 May 2017 / Published online: 14 November 2017

(C) The Author(s) 2017. This article is an open access publication

\begin{abstract}
The present work provides a formulation of a constitutive model for metals with the aim to simulate cyclic deformation under axial extension or compression assisted by cyclic torsional (or shearing) straining of specified amplitude and frequency. Such a mode of deformation was recently implemented in technological processes such as extrusion, forging and rolling, cf. Bochniak and Korbel (Eng Trans 47:351-367, 1999, J Mater Process Technol 134:120-134, 2003, Philos Mag 93:1883-1913, 2013, Mater Sci Technol 16:664-674, 2000). The constitutive model accounting for combined hardening (isotropic-kinematic) with both hardening and recovery effects is presented and calibrated for several materials: pure copper, aluminum alloy (2024), and austenitic steel. The experimental data are used to specify model parameters of materials tested, and next the cyclic response for different shear strain amplitudes is predicted and confronted with empirical data. The constitutive model is developed in order to simulate technological processes assisted by cyclic deformation.
\end{abstract}

\section{Introduction}

The cyclic deformational response of metals was extensively studied both experimentally and analytically by developing numerous constitutive models. The cyclic plasticity phenomena, such as Bauschinger effect, hardening and recovery, strain memory, ratcheting, amplitude dependent hardening, non-proportionality effect, were incorporated in model formulations, usually containing numerous material parameters. The plastic strain accumulation (ratcheting) for stress controlled cyclic loading imposed on mean stress is one of important phenomena requiring long-term study. In fact, for structural safety, the control of progressive strain accumulation is of fundamental importance. However, for cyclic strain controlled deformation imposed on progressive straining, the load reduction can be beneficial in executing the deformation process. In fact, in recent years, a growing interest is observed in metal forming processes assisted by cyclic loading. The so-called KOBO-type forming proposed by Korbel and Bochniak $[9-12,47]$ and applied to extrusion of tubes and wires has demonstrated essential advantages with respect to classical forming processes. The significant reduction of required load, growth of ductility, fine grain structure are main beneficial factors, cf. also Kong et al. [46].

The present work is aimed at the formulation of the constitutive model able to simulate quantitatively the cyclic material response for the case of progressive axial straining assisted by cyclic shear or torsional strains of

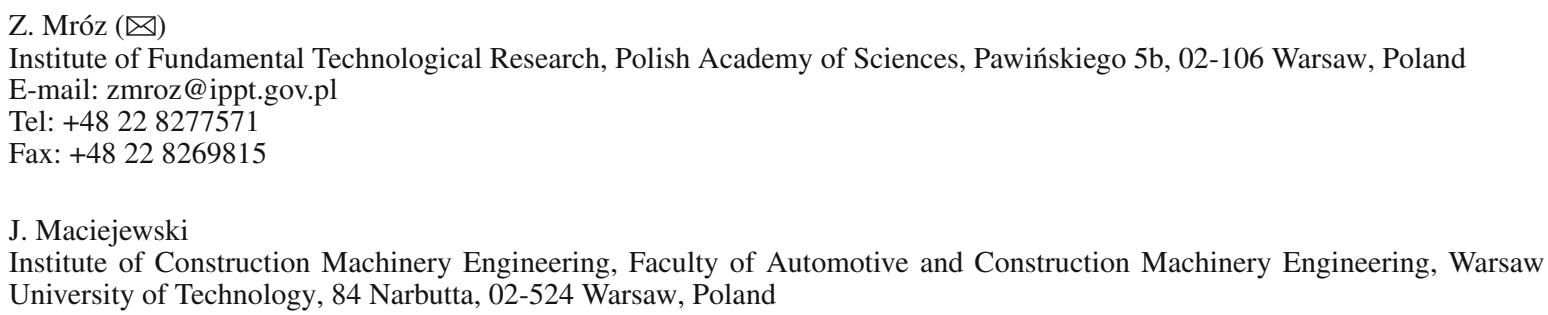


specified amplitude. The essential process parameters are then $\gamma_{m}$ and $\eta$ where $\gamma_{m}$ is the shear strain amplitude and $\eta$ is the ratio of shear and axial strain rates.

There have been numerous formulations of constitutive models aimed at the simulation of the inelastic response of metals for both proportional and non-proportional loading. Most models are based on the concept of interaction of hardening and dynamic recovery effects, with back stress increasing along the plastic strain rate orientation and decreasing proportionally to the back stress measure. In effect, the back stress evolves toward its limit value specified by the hardening saturation surface. The model originally proposed by Armstrong and Frederick [7] was next generalized by numerous researchers, cf. Burlet and Cailletaud [14], Moosbrugger and McDowell [56], Chaboche and Nouailhas [16,17], Chaboche [21], Jiang and Sehitoglu [35], Abdel and Ohno [5], Ohno and Kachi [61], Ohno [62], Chen et al. [23].

The multisurface hardening model formulation of Mróz [58] and generalized by Mróz and Rodzik [60] provides the translation rule of the yield surface with the back stress evolving toward its limit value on the consecutive hardening surface, thus accounting naturally for hardening and recovery effects. It was shown that the back stress evolution rules of those two classes of models are equivalent, cf. Chaboche and Rousellier [18], Lemaitre and Chaboche [53]. A notable success was achieved by developing simplified two-surface hardening models for which the variation of plastic hardening modulus was prescribed analytically, cf. Krieg [52] or Dafalias and Popov [29], Tseng and Lee [71], McDowell [55]. The multiple hardening surface concept was generalized by Chu [26] by assuming a continuous field of yield surfaces, thus providing hardening moduli. The numerical implementation of multisurface hardening plasticity was developed by Khoei and Jamali [44]. The concept of fuzzy set was applied by Klisinski [45] to simulate the cyclic response for a continuous field of loading surfaces.

The simplest hardening response in steady cyclic deformation has been based on the Masing rule for which the monotonic strain hardening curve of uniaxial-tension compression $\sigma=f(\varepsilon)$ is mapped onto the cyclic response $\Delta \sigma / 2=f(\Delta \varepsilon / 2)$, where $\Delta \sigma$ and $\Delta \varepsilon$ denote the stress and strain amplitudes. However, for nonproportional cyclic loading, the additional cyclic hardening is observed and is dependent on the form of the cyclic stress or strain path. However, the subsequent proportional cyclic deformation removes the accumulated hardening and restores the previous state reached in proportional loading. It should be noted that for large cyclic strain paths departing from proportionality, the hardening-recovery effect is observed due to microstructure evolution and generation of micro-shear bands, cf. Bouvier et al. [13], Kowalczyk-Gajewska et al. [48]. The effect of strain amplitude is also important as the steady hardening state depends on plastic strain amplitude and the material exhibits the memory of maximal amplitude reached in the loading process, cf. Trampczyński and Mróz [70] where the effect of strain amplitude and non-proportional hardening was studied and Ohno [63], where the review of modeling and experimental results of cyclic deformation has been presented. The extensive review of plastic and viscoplastic hardening models applicable for simulation of cyclic deformation was presented by Chaboche [19] and Kang [41].

Usually, for asymmetric stress amplitudes, the material exhibits a ratcheting effect for which the accumulation of plastic strain occurs and induces incremental failure. The quantitative simulation of ratcheting is a most difficult problem for the constitutive modeling of cyclic deformation, requiring a large number of material parameters. Most models were applied to simulate uniaxial and multiaxial strain ratcheting effects observed in stress or in mixed-control deformation programs. Let us mention such papers as Abdel-Karim and Ohno [5], Abdel-Karim [2-4,6], Abdel-Karim and Khan [1], Bari and Hassan [8], Burlet and Cailletaud [14], Chaboche [15,20-22], Chen et al. [24], Choi et al. [25], Feaugas and Gaudin [30], Hassan and Kyriakides [34], Hassan et al. [33], Jiang and Sehitoglu [35], Kang et al. [36-43], Khoei and Jamali [44], Moosbrugger and McDowell [56], Ohno[62,63], Ohno and Kachi [61], Portier et al. [66], Sai and Cailletaud [68], Tanaka et al. [69], Velay [72], Vincent et al. [74], Voyiadjis et al. [75], Yoshida et al. [76], Dafalias et al. [28,29], Feigenbaum and Dafalias [31], where an extensive literature can be found. In most models, the total back stress is assumed as a sum of several portions for which separate hardening-recovery evolutions rules are stated, similarly to the original formulation of Chaboche [21,22]. An interesting study of predictive capacity of selected constitutive models in simulation of multiaxial ratcheting strain was presented by Bari and Hassan [8] who used the experimental data of Hassan and Kyriakides [34] from thin-walled tubes subjected to internal pressure and tension. The ratcheting effects for combined tension-torsion loading were studied experimentally by Portier et al. [66] who also provided comparative analysis of predictions of several selected models. It was shown that for quantitative predictions of ratcheting strain, the number of material parameters increases and the model structure becomes complex. The experimental study of subsequent yield surfaces for loading-unloading programs was presented by Khan at al [42], and the material deformation response after large prestrain was discussed by Khan and Wang [43]. 
On the other hand, the fully strain controlled processes were not analyzed sufficiently in the literature. Instead of the plastic strain accumulation (ratcheting), we observe the monotonic stress reduction when the deformation process is assisted by cyclic straining. The analytical solution of tensile, or compressive deformation of cylinders or tubes of a perfectly plastic material with assistance of cyclic torsional strain was provided by Mróz et al. [57], where the steady-state stress paths and axial load reductions were discussed in detail. The related study of Kowalczyk-Gajewska et al. [48] provided the analysis of texture evolution affected by microshear bands developed in cyclic deformation process. The fine grain structure obtained in this process results from shear band and twin intersections including development of new grain boundaries and fine crystalline structure. The familiar technology of severe plastic deformation (SPD) to generate nanosize grain structure by cyclic non-proportional loading (such ECAP - equal channel angular pressing) certainly belongs to this class of problems, that is cyclic plasticity for kinematically induced large localized plastic straining, varying cyclically within the material element. The analysis of axisymmetric extrusion assisted by cyclic torsion was presented by Maciejewski and Mróz [54].

The aim of this paper is to present a cyclic hardening model with the aim to simulate quantitatively the material response under strain controlled cyclic loading in tension, torsion or in a combined mode of axial deformation with imposed cyclic torsion of specified shear strain amplitude. The model formulation is presented in Sect. 2 within the formalism of small strain theory. It has been calibrated for aluminum alloy 2024, medium carbon and austenitic steels, annealed copper, and next used to predict multiaxial deformation response. In Sect. 3, the model calibration, parameter sensitivity and application to simulate strain controlled processes are discussed in detail.

\section{Constitutive model formulation}

\subsection{Two-surface hardening-recovery model}

The combined effect of hardening and recovery during plastic deformation leads to attainment of the saturation state characterized by coaxiality of the back stress and plastic strain rate vectors. Consider first the kinematic hardening rule proposed by Armstrong and Frederick [7]. The yield condition and the flow rule are

$$
\begin{aligned}
f_{p} & =\sqrt{\frac{3}{2}(\boldsymbol{S}-\boldsymbol{X}) \cdot(\boldsymbol{S}-\boldsymbol{X})}-\sigma_{p}=0, \\
\dot{\boldsymbol{\varepsilon}}^{p} & =\dot{\lambda} \frac{\partial f_{p}}{\partial \boldsymbol{\sigma}}=\dot{\lambda} N=\dot{\lambda} \frac{\frac{3}{2}(\boldsymbol{S}-\boldsymbol{X})}{\sigma_{p}}, \dot{\lambda} \geq 0, \quad f_{p} \leq 0, \quad \dot{\lambda} f_{p}=0,
\end{aligned}
$$

where $\dot{\lambda}=\sqrt{\frac{2}{3} \dot{\boldsymbol{\varepsilon}}^{p} \cdot \dot{\boldsymbol{\varepsilon}}^{p}}, \boldsymbol{S}$ is the stress deviator, $\boldsymbol{S}=\boldsymbol{\sigma}-\frac{1}{3} \operatorname{tr} \boldsymbol{\sigma} 1, \boldsymbol{X}$ is the back stress tensor and $\sigma_{p}$ is the yield stress. Here, dot between two vectors or tensors denotes their scalar product and dot over a symbol denotes the rate with respect to a process evolution parameter. The back stress evolution is assumed in the form accounting for hardening and recovery:

$$
\dot{\mathbf{X}}=\frac{2}{3} c \dot{\boldsymbol{\varepsilon}}^{p}-\gamma \boldsymbol{X} \dot{\lambda}=\dot{\lambda}\left[\frac{c(\boldsymbol{S}-\boldsymbol{X})}{\sigma_{p}}-\gamma \boldsymbol{X}\right] .
$$

The saturation (limit state) is reached when

$$
\dot{\boldsymbol{X}}=0, \quad \text { or } \quad \frac{c}{\sigma_{p}}(\boldsymbol{S}-\boldsymbol{X})-\gamma \boldsymbol{X}=0
$$

and the saturation or limit state surface in the back stress space is expressed as

$$
F_{l}(\boldsymbol{X})=\sqrt{\frac{3}{2} \boldsymbol{X}_{l} \cdot \boldsymbol{X}_{l}}-r_{l}=0
$$

where $r_{l}=c / \gamma$. In the stress space the limit surface equation is

$$
F_{l}(\boldsymbol{\sigma})=\sqrt{\frac{3}{2} \boldsymbol{S}_{l} \cdot \boldsymbol{S}_{l}}-\sigma_{l}=0, \quad \sigma_{l}=\sigma_{p}+r_{l} .
$$




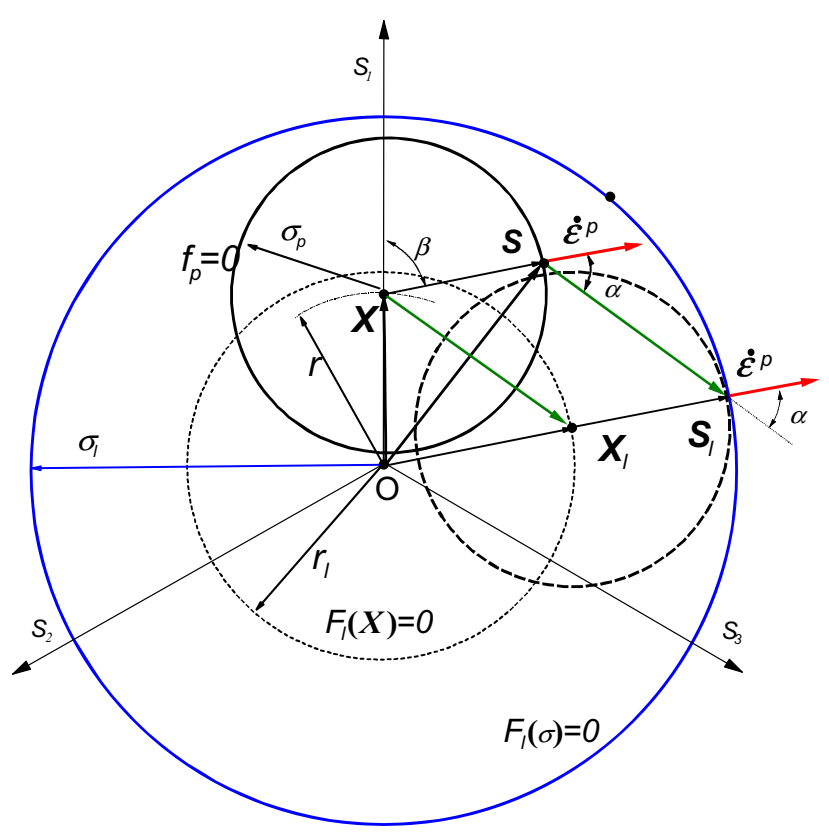

Fig. 1 Evolution of the back stress $\boldsymbol{X}$ to its saturation value $\boldsymbol{X}_{l}$

The back stress evolution rule (3) can now be rewritten in the form, cf. Fig. 1,

$$
\dot{\boldsymbol{X}}=\dot{\lambda} \gamma\left(\boldsymbol{S}_{\boldsymbol{l}}-\boldsymbol{S}\right)=\dot{\lambda} \gamma\left(\boldsymbol{X}_{\boldsymbol{l}}-\boldsymbol{X}\right)=\dot{\lambda} \gamma \rho \Delta,
$$

where $S_{l}$ and $X_{l}$ are the saturation states associated with the instantaneous plastic strain rate orientation, thus

$$
\frac{S_{l}}{\left\|S_{l}\right\|}=\frac{X_{l}}{\left\|X_{l}\right\|}=\frac{\dot{\boldsymbol{\varepsilon}}^{p}}{\left\|\dot{\boldsymbol{\varepsilon}}^{p}\right\|}
$$

and

$$
\begin{aligned}
& \boldsymbol{S}_{\boldsymbol{l}}=(\boldsymbol{S}-\boldsymbol{X})\left(1+\frac{r_{l}}{\sigma_{p}}\right)=(\boldsymbol{S}-\boldsymbol{X}) \frac{\sigma_{l}}{\sigma_{p}}, \quad \boldsymbol{S}_{l}-\boldsymbol{S}=\boldsymbol{X}_{l}-\boldsymbol{X}=\boldsymbol{S} \frac{r_{l}}{\sigma_{p}}-\boldsymbol{X} \frac{\sigma_{l}}{\sigma_{p}}, \\
& \boldsymbol{X}_{\boldsymbol{l}}=(\boldsymbol{S}-\boldsymbol{X}) \frac{r_{l}}{\sigma_{p}}, \quad \boldsymbol{\rho}=\frac{\boldsymbol{S}_{\boldsymbol{l}}-\boldsymbol{S}}{\left\|\boldsymbol{S}_{\boldsymbol{l}}-\boldsymbol{S}\right\|}
\end{aligned}
$$

$$
\Delta=\left|S_{l}-S\right|=\left|X_{l}-X\right|=\sqrt{\frac{3}{2}\left(S_{l}-S\right) \cdot\left(S_{l}-S\right)}=\sqrt{\frac{3}{2}\left(X_{l}-X\right) \cdot\left(X_{l}-X\right)}=\sqrt{r_{l}^{2}+r^{2}-2 r r_{l} \cos \beta}
$$

where $r=\sqrt{\frac{3}{2} \boldsymbol{X} \cdot \boldsymbol{X}}$ and $\beta$ is the angle between $\boldsymbol{O X}$ and $\dot{\boldsymbol{\varepsilon}}^{p}$ or $(\boldsymbol{S}-\boldsymbol{X})$. Note that the vector norm is scaled by $\sqrt{3 / 2}$, and the vector $\rho$ satisfies the relation $\sqrt{\frac{3}{2} \rho \cdot \rho}=1$

Considering a deformation process with constant orientation of the plastic strain rate vector, the back stress $\boldsymbol{X}$ tends to its limiting value $\boldsymbol{X}_{\boldsymbol{l}}$ coaxial with the plastic strain trajectory for specified $\dot{\boldsymbol{\varepsilon}}^{p}$ of constant orientation. In fact, $\boldsymbol{X}_{\boldsymbol{l}}$ can be treated as the end of the radial stress path associated with the proportional plastic deformation. The instantaneous back stress state $\boldsymbol{X}$ evolves to its radial limit value $\boldsymbol{X}_{\boldsymbol{l}}$ and next remains fixed for the proportional deformation. Let us note that this translation rule is identical to that proposed originally by Mróz [58] in formulating the multisurface hardening model. The active nested loading surface has been assumed to translate toward the image stress point on the consecutive surface according to the rule (7).

The hardening modulus $H$ can be expressed from the consistency condition. Neglecting the corotational stress and back stress rates, it can be written as

$$
\dot{f}_{p}=\frac{\partial f_{p}}{\partial \boldsymbol{\sigma}} \cdot \dot{\boldsymbol{\sigma}}+\frac{\partial f_{p}}{\partial \boldsymbol{X}} \cdot \dot{\boldsymbol{X}}=\frac{\partial f_{p}}{\partial \boldsymbol{\sigma}} \cdot \dot{\boldsymbol{\sigma}}-\frac{\partial f_{p}}{\partial \boldsymbol{\sigma}} \cdot \dot{\boldsymbol{X}}=0,
$$




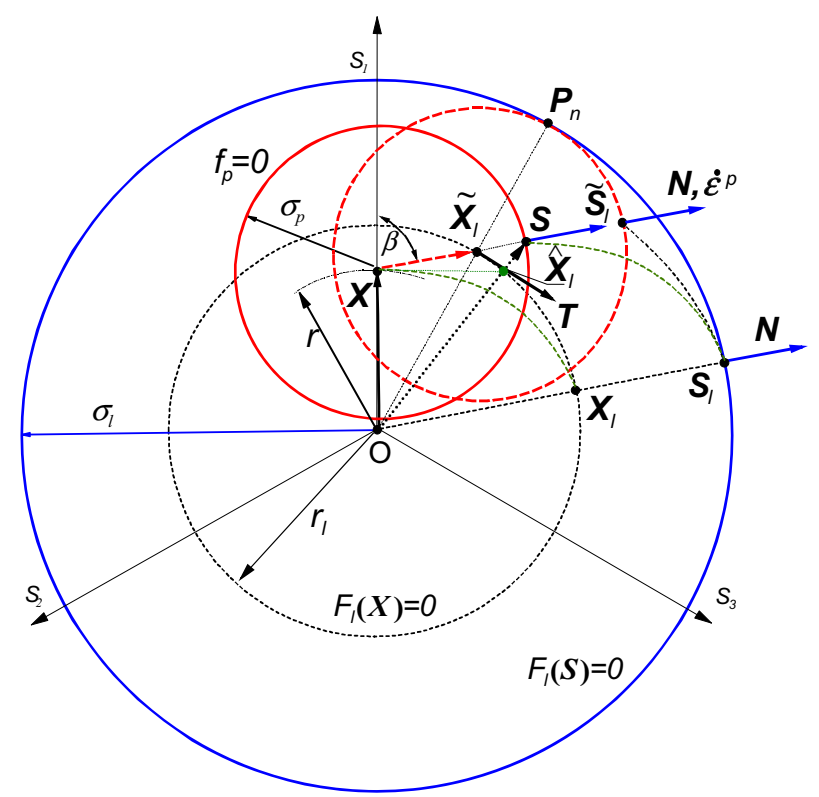

Fig. 2 Evolution of the back stress $\boldsymbol{X}$ to its limit value $\tilde{\boldsymbol{X}}_{l}$ following Prager translation rule and subsequent reorientation process to the coaxial state $\boldsymbol{X}_{l}$

and in view of (7) and (9), we have

$$
\begin{aligned}
\dot{\lambda} H & =\frac{\partial f_{p}}{\partial \boldsymbol{\sigma}} \cdot \dot{\boldsymbol{\sigma}}, \quad H=\gamma \frac{\partial f_{p}}{\partial \boldsymbol{\sigma}} \cdot\left(\boldsymbol{S}_{\boldsymbol{l}}-\boldsymbol{S}\right)=c-\frac{3}{2} \gamma\left(\boldsymbol{X} \cdot \frac{\boldsymbol{S}-\boldsymbol{X}}{\sigma_{p}}\right) \\
& =\gamma\left(r_{l}-r \cos \beta\right)=c\left(1-\frac{r}{r_{l}} \cos \beta\right)=\frac{1}{2} c\left[1-\left(\frac{r}{r_{l}}\right)^{2}+\left(\frac{\Delta}{r_{l}}\right)^{2}\right] .
\end{aligned}
$$

Relations (11) specify the field of hardening moduli for states within the limit surface. For the proportional loading we have $\beta=0, H=c-\gamma r$, for transverse loading after initial prestrain there is $\beta=\pi / 2, H=c$ and for proportional unloading $\beta=\pi, H=c+\gamma r$. The hardening modulus thus depends on the orientation of plastic strain rate with respect to the initial prestrain.

The present model predicts the coaxiality of the plastic strain rate and the back stress $\boldsymbol{X}$ in the limit state. The stress states in the exterior of the limit surfaces are not allowed. However, the Melan-Prager translation rule can be formulated by assuming the back stress evolution following plastic strain rate vector, thus

$$
\dot{X}=\dot{\lambda} \frac{2}{3} c\left(1-\frac{r}{r_{l}} \cos \beta\right) N, \quad r \leq r_{l}
$$

From the consistency condition (10), it follows that hardening modulus $H$ now equals

$$
H=c\left(1-\frac{r}{r_{l}} \cos \beta\right), \quad r \leq r_{l} .
$$

Referring to Fig. 2, it is seen that $\boldsymbol{X}$ evolves toward $\tilde{\boldsymbol{X}}_{\boldsymbol{l}}$ specified by the orientation of plastic strain rate vector, so that

$$
\tilde{\boldsymbol{X}}_{\boldsymbol{l}}=\boldsymbol{X}+\mu(\boldsymbol{S}-\boldsymbol{X}), \quad F_{l}\left(\boldsymbol{X}_{l}\right)=\sqrt{\frac{3}{2} \boldsymbol{X}_{l} \cdot \boldsymbol{X}_{l}}-r_{l}=0,
$$

where the scalar factor $\mu$ is determined from (14), thus

$$
\mu=\sqrt{\left(\frac{r_{l}}{\sigma_{p}}\right)^{2}-\left(\frac{r}{\sigma_{p}}\right)^{2} \sin ^{2} \beta}-\frac{r}{\sigma_{p}} \cos \beta .
$$


Let us note that the backstress $\tilde{\boldsymbol{X}}_{l}$ is not coaxial with $\dot{\boldsymbol{\varepsilon}}^{p}$. The coaxiality can be attained by consecutive backstress reorientation process when $\tilde{\boldsymbol{X}}_{l}$ moves along the limit surface $F_{l}=0$ to the limit coaxial state $\boldsymbol{X}_{l}$, Fig. 2. Assume this evolution in the form

$$
\dot{\boldsymbol{X}}=\dot{\lambda} \frac{2}{3} c\left(1-\frac{r}{r_{l}} \cos \beta\right) \boldsymbol{T}, \quad \boldsymbol{T}=\boldsymbol{N}\left(\boldsymbol{I}-\boldsymbol{x}_{l} \otimes \boldsymbol{x}_{l}\right), \quad r \leq r_{l}
$$

where $\boldsymbol{T}$ is the tangent vector to the limit surface $F_{l}=0$ and $\boldsymbol{x}_{l}=\boldsymbol{X}_{l} \|_{\left\|\boldsymbol{X}_{l}\right\|}$ is the unit vector specifying the back stress orientation. From the consistency condition (10), it follows that $H=c \sin ^{2} \beta$ and the modulus $H$ vanishes when $\tilde{\boldsymbol{X}}_{l}$ coincides with $\boldsymbol{X}_{l}$ since then $\beta=0$.

The evolution rules (12) and (16) specify the back stress path $\boldsymbol{X}-\tilde{\boldsymbol{X}}_{l}-\boldsymbol{X}_{l}$, first along the orientation $\boldsymbol{N}$ next on the limit surface to the coaxial state $\boldsymbol{X}_{l}$. The rule (7) specifies the direct evolution $\boldsymbol{X}-\boldsymbol{X}_{l}$ to the coaxial state. The intermediate paths of the evolution toward $\boldsymbol{X}_{l}$ can be generated by assuming the back stress to evolve toward the state $\hat{\boldsymbol{X}}_{l}$ on the limit surface, coaxial with the stress vector $S$, thus

$$
\dot{\boldsymbol{X}}=\dot{\lambda} \gamma\left(\hat{\boldsymbol{X}}_{l}-\boldsymbol{X}\right)=\dot{\lambda} \gamma\left(S \frac{r_{l}}{s}-\boldsymbol{X}\right), \quad s=\sqrt{\frac{3}{2} \boldsymbol{S} \cdot \boldsymbol{S}} .
$$

During plastic straining with constant orientation $N$, the point $\hat{\boldsymbol{X}}_{l}$ evolves toward $\boldsymbol{X}_{l}$ and the curvilinear back stress trajectory reaches the limit state $\boldsymbol{X}_{l}$. The evolutions rules proposed by Ohno and Wang [64] and Ohno [62] predict also curvilinear back stress path tending to the limit state. A different evolution rule was proposed by Burlet and Cailletaud [14], who postulated the back stress evolution to follow the orientation of $N$ toward the non-coaxial limit point $\tilde{X}_{l}$ and next to continue for inducing softening response. These rules could be useful in a simulation of ratcheting strain accumulation for multiaxial non-proportional cyclic loading. The comparative analysis of predictive performance of several back stress evolution rules was recently presented by Abdel-Karim [2]. In the present work, the rule (7) will be applied; however, the limit surface will be allowed to expand and translate, thus affecting the back stress evolution.

For the uniaxial loading and unloading, we have

$$
\begin{aligned}
& f_{p}=\left|\sigma-X^{\prime}\right|-\sigma_{p}=0, \\
& \dot{X}^{\prime}=\frac{2}{3} c \dot{\boldsymbol{\varepsilon}}^{p}-\boldsymbol{X}^{\prime}\left|\dot{\boldsymbol{\varepsilon}}^{p}\right|,
\end{aligned}
$$

where $X^{\prime}=3 / 2 X$ (projection of the $X_{1}$ back stress component on the $\sigma_{1}$ axis). Integrating (3) or (7), we obtain

$$
X^{\prime}=\mu \frac{c}{\gamma}+\left(X_{0}^{\prime}-\mu \frac{c}{\gamma}\right) e^{-\mu \gamma\left(\varepsilon^{p}-\varepsilon_{0}^{p}\right)},
$$

where $\mu=1$ for loading, $\mu=-1$ for unloading and $X_{0}^{\prime}, \varepsilon_{0}^{p}$ are the initial values. For $X_{0}^{\prime}=\varepsilon_{0}^{p}=0$, we have

$$
X^{\prime}=\mu \frac{c}{\gamma}\left(1-e^{-\mu \gamma \varepsilon^{p}}\right)
$$

Figure 3 presents the material response for uniaxial cyclic stress, Fig. 3a, and for the cyclic stress or strain imposed on the initial prestrain. It is seen that there is mean stress relaxation for the specified strain amplitude of imposed cyclic straining, Fig. 3b, or excessive ratcheting effect for specified cyclic stress amplitude and mean stress value, Fig. 3c.

\subsection{Generalized two-surface hardening-recovery model}

The present generalization follows the multisurface hardening model formulation where instead of the limit surface a set of hardening surfaces is introduced, cf. Mróz [58] or Mróz and Rodzik [60]. The translation of the yield surface is assumed to follow the distance vector $\boldsymbol{S}_{\boldsymbol{l}}-\boldsymbol{S}$ and the relations (7)-(9) apply. However, now the surface $F_{l}=0$ is not the limit surface but the hardening surface which may expand or expand and translate in the course of plastic straining. The model equations are formulated by assuming only one or two hardening surfaces and a nonlinear back stress evolution rule, in a way similar to that proposed for two-surface plasticity models, cf. Krieg [52] or Dafalias and Popov [29]. 


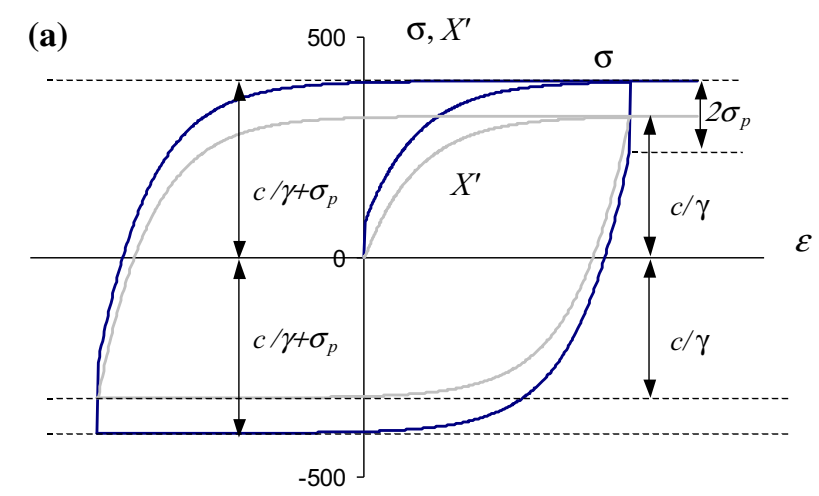

(b)

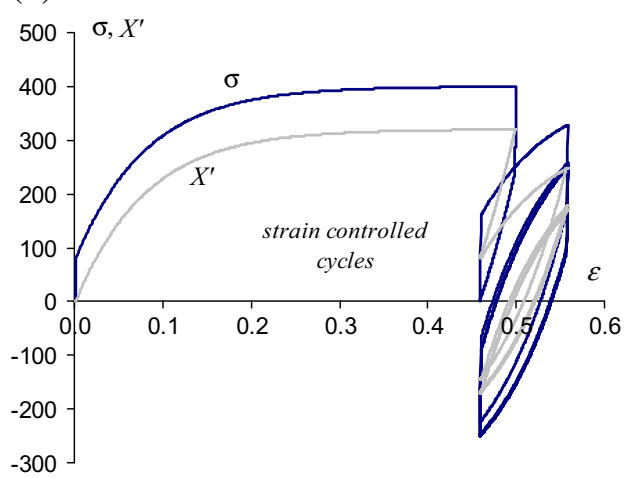

(c)

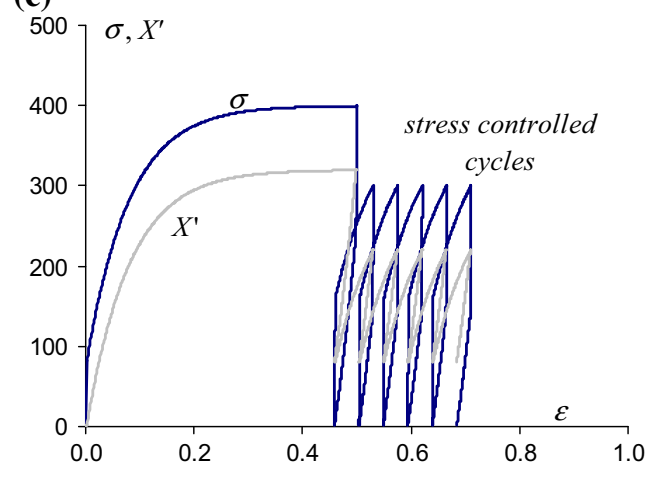

Fig. 3 Loading-unloading response in the uniaxial case; a symmetric strain controlled cycles; b strain controlled cycles after initial prestrain; c stress controlled cycles after initial prestrain

Consider first the isotropic expansion of the hardening surface $F_{l}=0$, dependent on the amplitude of cyclic stress. Similarly, as previously mentioned, we have

$$
\begin{aligned}
f_{p} & =\sqrt{\frac{3}{2}(\boldsymbol{S}-\boldsymbol{X}) \cdot(\boldsymbol{S}-\boldsymbol{X})}-\sigma_{p}(\xi)=0, \\
F_{l}(\boldsymbol{S}) & =\sqrt{\frac{3}{2} \boldsymbol{S}_{l} \cdot \boldsymbol{S}_{l}}-\sigma_{l}(\xi)=0 \text { or } F_{l}(\boldsymbol{X})=\sqrt{\frac{3}{2} \boldsymbol{X}_{l} \cdot \boldsymbol{X}_{l}}-r_{l}(\xi) .
\end{aligned}
$$

Assuming that there is no isotropic hardening effect for $l \leq l_{0}$, we can write

$$
\dot{\xi}= \begin{cases}\dot{\lambda}\left(\frac{l-l_{0}}{1-l_{0}}\right)^{\kappa}=\dot{\lambda} L^{\kappa} & l>l_{0}, \\ 0 & l \leq l_{0},\end{cases}
$$

where $\dot{\lambda}=\dot{\varepsilon}^{e f}=\sqrt{\frac{2}{3} \dot{\varepsilon}^{p} \cdot \dot{\boldsymbol{\varepsilon}}^{p}}$, and

$$
\begin{gathered}
l=1-\frac{|\Delta|}{2\left(\sigma_{l}-\sigma_{p}\right)}=1-\frac{\Delta}{\Delta_{\max }}=1-\frac{\sqrt{\frac{3}{2}\left(\boldsymbol{S}_{l}-\boldsymbol{S}\right) \cdot\left(\boldsymbol{S}_{l}-\boldsymbol{S}\right)}}{2\left(\sigma_{l}-\sigma_{p}\right)}, \\
\Delta=|A B|=\left|\boldsymbol{S}_{l}-\boldsymbol{S}\right|=\sqrt{\frac{3}{2}\left(\boldsymbol{S}_{l}-\boldsymbol{S}\right) \cdot\left(\boldsymbol{S}_{l}-\boldsymbol{S}\right)}, \quad \Delta_{\max }=2\left(\sigma_{l}-\sigma_{p}\right),
\end{gathered}
$$

In the following, we assume that both yield and hardening surfaces expand, but the ratio of their diameters is constant, thus

$$
k_{p}=\frac{\sigma_{l}}{\sigma_{p}}=\text { const. }
$$


The isotropic hardening rule is assumed in a form

$$
\sigma_{l}=\sigma_{l 0}+s \xi^{w}
$$

or

$$
\sigma_{l}=\sigma_{l 0}+\left(\sigma_{l f}-\sigma_{l 0}\right)\left(1-e^{-w \xi^{s}}\right),
$$

where $\sigma_{l 0}$ is the initial radius of the hardening surface and $\sigma_{l f}$ is the asymptotic value, $s$ and $w$ are the hardening parameters. The parameters $s, w$ are assumed as constant.

Let us note that the rule (25) predicts unlimited growth of $\sigma_{l}$ for increasing $\xi$ but the rule (26) predicts evolution toward the limit value $\sigma_{l f}$. Thus the rule (25) can be applied to simulate the initial transient hardening period but not the saturated hardening state.

The hardening modulus is now specified from the consistency condition

$$
\begin{aligned}
\dot{f}_{p} & =\boldsymbol{N} \cdot \dot{\boldsymbol{\sigma}}-\boldsymbol{N} \cdot \dot{\boldsymbol{X}}-\sigma_{p}^{\prime} \dot{\xi}=0, \\
\boldsymbol{N} & =\frac{\partial f_{p}}{\partial \boldsymbol{\sigma}}=\frac{\frac{3}{2}(\boldsymbol{S}-\boldsymbol{X})}{\sigma_{p}},
\end{aligned}
$$

and in view of (7), we have

$$
\dot{\lambda}=\frac{N \cdot \dot{\sigma}}{H}, \quad H=\gamma N \cdot\left(S_{l}-S\right)+\sigma_{p}^{\prime} L^{\kappa},
$$

where $\sigma_{p}^{\prime}=\partial \sigma_{p} / \partial \xi$. From (21) and (22), we obtain

$$
H=\gamma \Delta \cos \alpha+\sigma_{p}^{\prime} L^{\kappa}=c\left(1-\frac{r}{r_{l}} \cos \beta\right)+\sigma_{p}^{\prime} L^{\kappa},
$$

where $\alpha$ is the angle between the vectors $S_{l}-S$ and $N$ and $\Delta$ denotes the distance of the stress point to the radially mapped point on the limit surface. We have

$$
\begin{aligned}
\Delta & =\left|\boldsymbol{S}_{\boldsymbol{l}}-\boldsymbol{S}\right|=\sqrt{\frac{3}{2}\left(\boldsymbol{S}_{\boldsymbol{l}}-\boldsymbol{S}\right) \cdot\left(\boldsymbol{S}_{\boldsymbol{l}}-\boldsymbol{S}\right)}=\sqrt{\frac{3}{2}\left[k_{p}(\boldsymbol{S}-\boldsymbol{X})-\boldsymbol{S}\right] \cdot\left[k_{p}(\boldsymbol{S}-\boldsymbol{X})-\boldsymbol{S}\right]} \\
& =\sqrt{\sigma_{p}^{2}\left(k_{p}-1\right)^{2}+r^{2}-2 \sigma_{p}\left(k_{p}-1\right) r \cos \beta}, \quad r=|\boldsymbol{X}|=\sqrt{\frac{3}{2} \boldsymbol{X} \cdot \boldsymbol{X}} .
\end{aligned}
$$

Let us note that the values of $\Delta$ for $\beta=0, \pi / 2$ and $\pi$ are equal to

$$
\begin{array}{llll} 
& \text { Proportional loading: } & \beta & =0: \quad \Delta(0)=\sigma_{p}\left(k_{p}-1\right)-r \\
\text { Transverse loading: } & \beta & =\pi / 2: \Delta\left(\frac{\pi}{2}\right)=\sqrt{\sigma_{p}^{2}\left(k_{p}-1\right)^{2}+r^{2}} \\
\text { Unloading and reverse loading: } & \beta=\pi: & \Delta(\pi)=\sigma_{p}\left(k_{p}-1\right)+r
\end{array}
$$

and $\Delta_{\max }=2\left(\sigma_{l}-\sigma_{p}\right)=2 \sigma_{p}\left(k_{p}-1\right)$

Consider now the proportional loading path, $\beta=\alpha=0$, and assume the asymptotic value of hardening modulus $H=H_{l}$. Assuming $\kappa=1, l_{0}=0, L=l$ from (29) it follows that

$$
H=\gamma \Delta \cos \alpha+\sigma_{p}^{\prime} l=\gamma \Delta_{l}+\sigma_{p}^{\prime}\left(1-\frac{\Delta_{l}}{\Delta_{\max }}\right)=H_{l}
$$

and

$$
\Delta_{l}=\frac{H_{l}-\sigma_{p}^{\prime}}{\gamma-\sigma_{p}^{\prime} / \Delta_{\max }}
$$

is the limit distance of the yield and hardening surfaces. For $\sigma_{p}^{\prime}=0$, we have $\gamma \Delta_{l}=H_{l}$. The distance parameters $l$ now equals

$$
l=1-\frac{\Delta}{\Delta_{\max }}=1-\frac{\sqrt{\sigma_{p}^{2}\left(k_{p}-1\right)^{2}+r^{2}-2 \sigma_{p}\left(k_{p}-1\right) r \cos \beta}}{2 \sigma_{p}\left(k_{p}-1\right)}
$$


and the angles $\alpha$ and $\beta$ are interrelated, thus

$$
\cos \alpha=\frac{\sigma_{p}\left(k_{p}-1\right)-r \cos \beta}{\Delta} .
$$

The alternative rules of hardening could be postulated as follows:

$$
\dot{X}=f(l) \rho \dot{\lambda}, \quad \rho=\frac{S_{l}-S}{\left\|S_{l}-S\right\|},
$$

where $\rho$ is the normalized vector along the path $S_{l}-S$

Now we have

$$
H=f(l) \cos \alpha+\sigma_{p}^{\prime} L^{\kappa} .
$$

The hardening function can be assumed in the form interpolating the hardening modulus between the initial and limit values, thus

$$
f(l)=H_{0}-\left(H_{0}-H_{l}\right) l^{p}
$$

and $f(l)=H_{0}$ for $l=0$ and $H=H_{l}$ for $l=l$.

Figure 4a presents the evolution of $\sigma_{l}$ corresponding to two rules (25) and (26). Figure $4 \mathrm{~b}$ presents the stress-strain curve and evolution of back stress $X^{\prime}$ and $l$ in the loading-unloading process of pure copper for large uniaxial straining. The experimental points, obtained by Follansbee and Kocks [32] are also introduced in Fig. 4a, b.

The weighting parameter $l$ depends on the distance of the stress point to the hardening surface. When the yield surface approaches the hardening surface, then $l \rightarrow 1$, but for the other cases there is $0 \leq l \leq 1$. Thus, the deformation paths more distant from the hardening surface induce lower hardening than the paths approaching the surface $F_{l}=0$. Similarly, the cycles of lower stress or strain amplitudes correspond to lower hardening rates than the cycles of higher amplitudes. Figure 5 presents the evolution of hysteresis loops for three strain amplitudes, assuming $\kappa=10, l_{0}=0$. The stress amplitude evolves toward the steady state with the rate of evolution much smaller for smaller strain amplitudes, cf. Fig 5b. In this way we incorporate the effect of amplitude dependent hardening rate in the cyclic deformation process.

\subsection{Three-surface hardening-recovery model}

Consider now a more general model for which the evolution of back stress $\boldsymbol{X}$ is affected by the second microlevel back stress $\boldsymbol{Y}$ inducing additional hardening. The evolution rule (3) now has the form

$$
\dot{\boldsymbol{X}}=\dot{\lambda}\left[\frac{c(\boldsymbol{S}-\boldsymbol{X})}{\sigma_{p}}-\gamma(\boldsymbol{X}-\boldsymbol{Y})\right]
$$
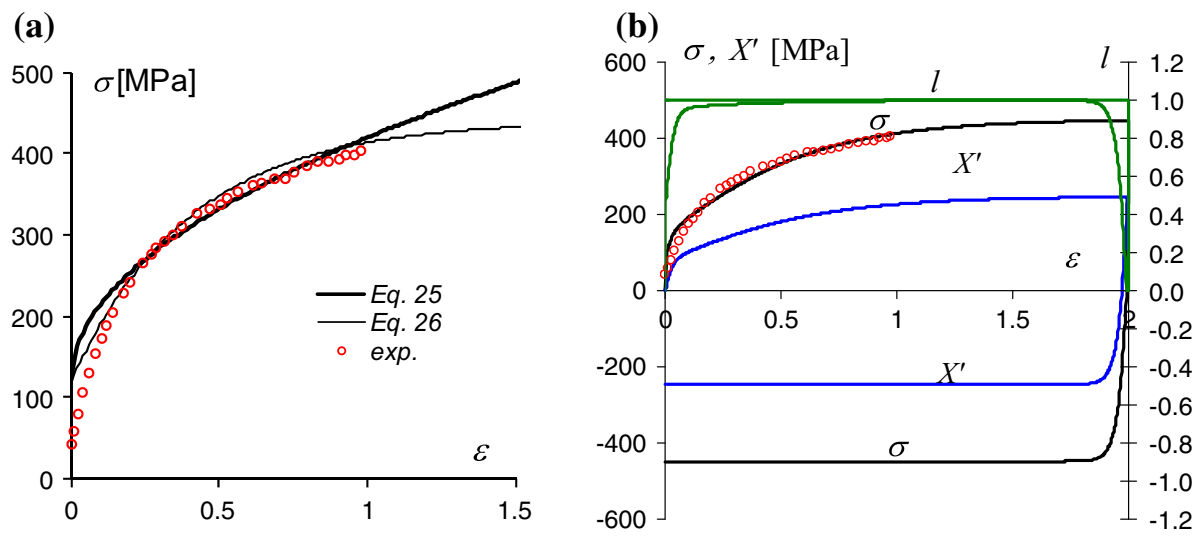

Fig. 4 Annealed copper: a Evolution of the limit stress $\sigma_{l}$, b the stress-strain curve for monotonic and reverse straining with the evolution of back stress and distance parameter $l$ 
and the saturation state $\dot{\boldsymbol{X}}=0$ is attained on the hardening surface, which is allowed to translate and expand. In view of (39), the hardening surface equation is now specified in the form

$$
F_{h}=\sqrt{\frac{3}{2}\left(\boldsymbol{S}_{l}-\boldsymbol{Y}\right) \cdot\left(\boldsymbol{S}_{l}-\boldsymbol{Y}\right)}-\sigma_{l}(\xi)=0 \quad \text { or } \quad F_{h}=\sqrt{\frac{3}{2}\left(\boldsymbol{X}_{l}-\boldsymbol{Y}\right) \cdot\left(\boldsymbol{X}_{l}-\boldsymbol{Y}\right)}-r_{l}(\xi)=0
$$

and its translation rule is expressed by assuming that $\boldsymbol{Y}$ tends to $\boldsymbol{X}$ for small value of its scalar norm or tends to a convergence state $\boldsymbol{Y}_{\boldsymbol{l}}$ coaxial with $\boldsymbol{X}$ for a larger norm value. It can be written

$$
\begin{aligned}
\dot{\boldsymbol{Y}}=\dot{\lambda} \gamma_{l}\left(\boldsymbol{Y}_{\boldsymbol{l}}-\boldsymbol{Y}\right) & \text { for } r=\sqrt{\frac{3}{2} \boldsymbol{X} \cdot \boldsymbol{X}}>R_{l}, \\
\dot{\boldsymbol{Y}}=\dot{\lambda} \gamma_{l}(\boldsymbol{X}-\boldsymbol{Y}) & \text { for } r \leq R_{l},
\end{aligned}
$$

where $\gamma_{l}$ is the material parameter and $\boldsymbol{Y}_{l}$ is the limit convergence state. The constitutive model of hardening with multilevel back stress interaction was discussed by Mróz and Rodzik [60]. The integral representation of back stress evolution was also considered and compared with the multisurface model predictions. The present formulation follows some assumptions of this model. It can be interpreted that $\boldsymbol{X}$ represents the residual stress at the grain scale and $\boldsymbol{Y}$ is the residual stress at the crystalline lattice scale induced by varying dislocation pattern. Assume, as previously mentioned that $\boldsymbol{Y}_{l}$ lies on the limit surface whose equation is of the form

$$
F_{y}=\sqrt{\frac{3}{2} \boldsymbol{Y}_{l} \cdot \boldsymbol{Y}_{l}}-R_{l}(\xi)=R-R_{l}=0,
$$

where $R_{l}$ is the limit surface radius. Similarly as previously we assume that both limit and hardening surfaces expand, but the ratio of their diameters is constant, thus

$$
k_{l}=\frac{\sigma_{l}}{R_{l}}=\text { const. }
$$

Let us note that when $r \leq R_{l}$, the relation (39) can be expressed as follows:

$$
\dot{X}=\frac{2}{3} c \dot{\varepsilon}^{p}-\frac{\gamma}{\gamma_{l}} \dot{Y}
$$

and for the case of proportional loading, like cyclic tension-compression, after integration we have

$$
X+\frac{\gamma}{\gamma_{l}} Y=\frac{2}{3} c \varepsilon^{p}+\text { const. }
$$

It follows from (45) that when the back stress $\boldsymbol{X}$ is represented by a point lying in the interior domain specified by the limit surface $F_{y}=0$, it can be expected that in the steady state, the ratcheting effect does not occur since the plastic strain is explicitly expressed in terms of varying periodically back stresses $\boldsymbol{X}$ and $\boldsymbol{Y}$.
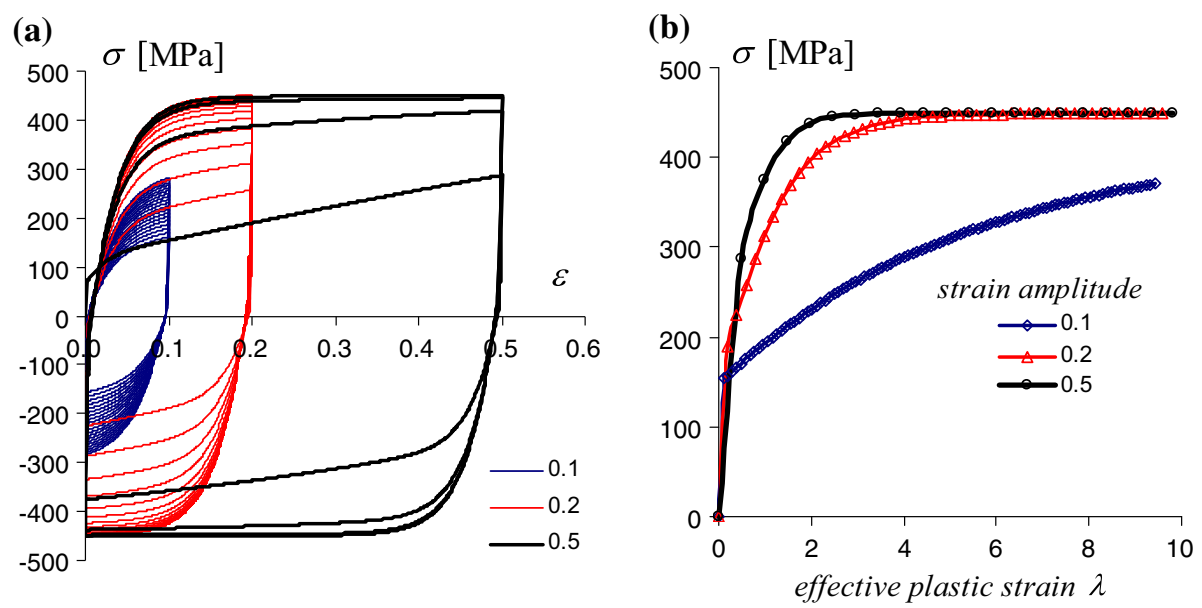

Fig. 5 Stress-strain response in the cyclic tension-compression test for various amplitudes: a evolution to steady states for specified amplitudes $\varepsilon_{\mathrm{a}}=0.1,0.2,0.5, \mathbf{b}$ evolution of the maximal stress in cyclic hardening process 


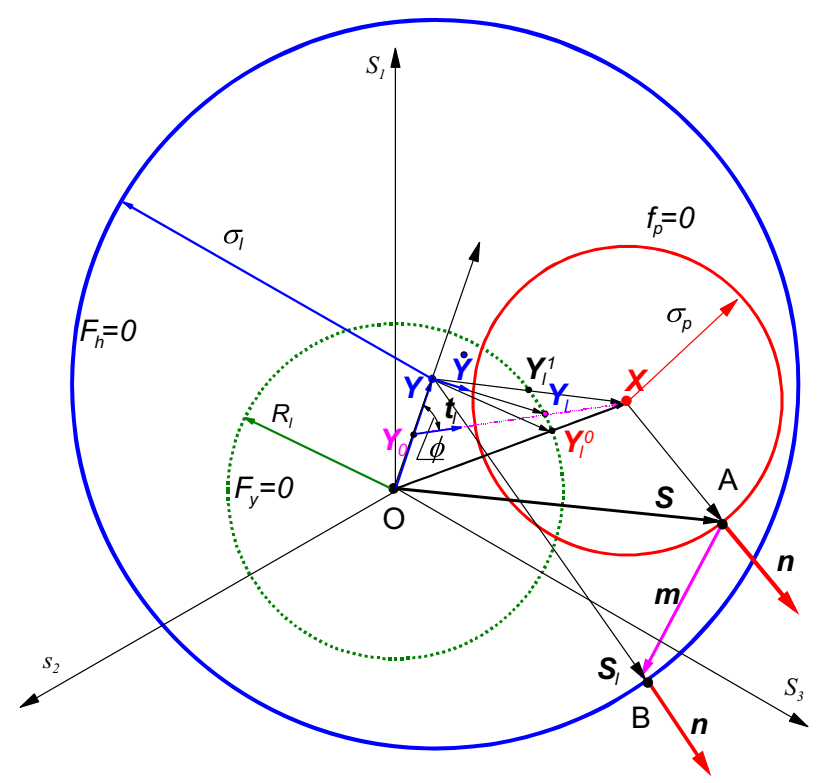

Fig. 6 Three-surface hardening model: evolution of the back stress $\boldsymbol{Y}$ toward the limit point $\boldsymbol{Y}_{l}$

A simple evolution rule is obtained by assuming that $\boldsymbol{Y}$ tends to the radial state $\boldsymbol{Y}_{l}^{0}$ on the limit surface coaxial with the back stress $\boldsymbol{X}$, so that

$$
Y_{l}^{0}=X \frac{R_{l}}{R}
$$

This assumption is identical to that taken in the evolution rule (9) for the back stress $\boldsymbol{X}$, which is now regarded as an active microstress inducing the evolution of $\boldsymbol{Y}$ toward a coaxial state. The other assumption is that $\boldsymbol{Y}$ follows the path $\boldsymbol{X}-\boldsymbol{Y}$ and reaches the limit point $\boldsymbol{Y}_{l}^{l}$, Fig. 6. We may generalize these two assumptions by postulating that $\boldsymbol{Y}_{l}$ is specified by the vector $\boldsymbol{X}-\boldsymbol{Y}_{0}$, where $\boldsymbol{Y}_{0}=f \boldsymbol{Y}, 0 \leq f \leq 1$, so we have

$$
\boldsymbol{Y}_{l}=\boldsymbol{Y}_{0}+\boldsymbol{t}_{l} \rho, \quad \boldsymbol{t}_{l}=\frac{\boldsymbol{X}-\boldsymbol{Y}_{\boldsymbol{0}}}{\left\|\boldsymbol{X}-\boldsymbol{Y}_{\boldsymbol{0}}\right\|} .
$$

Here, $\boldsymbol{t}_{l}$ is the unit vector along $\boldsymbol{X}-\boldsymbol{Y}_{\boldsymbol{0}}$ and the scalar factor $\rho$ is specified from the limit state condition, thus

$$
F_{y}=\sqrt{\frac{3}{2} \boldsymbol{Y}_{l} \cdot \boldsymbol{Y}_{l}}-R_{l}=\sqrt{\frac{3}{2}\left(\boldsymbol{Y}_{0}+\boldsymbol{t}_{l} \rho\right) \cdot\left(\boldsymbol{Y}_{0}+\boldsymbol{t}_{l} \rho\right)}-R_{l}=0,
$$

Equation (47) provides the value of the scalar factor $\rho$, namely

$$
\rho=-R_{0} \cos \phi+\sqrt{R_{l}^{2}-R_{0}^{2} \sin ^{2} \phi}, \quad R_{0}=\sqrt{\frac{3}{2} \boldsymbol{Y}_{0} \cdot \boldsymbol{Y}_{0}}=f R, \quad \cos \phi=\frac{\boldsymbol{Y} \cdot \boldsymbol{t}_{l}}{\|\boldsymbol{Y}\|},
$$

where $\phi$ is the angle between $\boldsymbol{O Y}$ and $\boldsymbol{X}-\boldsymbol{Y}_{0}$.

Let us note that when $f=0$, the limit state $\boldsymbol{Y}_{l}^{0}$ is specified by (46), when $f=1$ the state $\boldsymbol{Y}_{l}^{l}$ is generated. The fraction factor $f$ is identified from the multiaxial ratcheting tests, for instance from combined monotonic tension-cyclic torsion tests. It turns out that the position of the limit state $\boldsymbol{Y}_{l}$ is very essential in accurate prediction of the ratcheting strain. In fact, it controls the evolution path of the back stress $\boldsymbol{Y}$ to the coaxial state $\boldsymbol{Y}_{l}^{0}$. When the back stress $\boldsymbol{X}$ is represented by the point inside the limit surface $F_{y}=0$, then according to (41) we set $\boldsymbol{Y}_{l}=\boldsymbol{X}$.

Figure 6 illustrates the evolution rule of the back stress $\boldsymbol{Y}$. The hardening modulus now equals

$$
H=\gamma \boldsymbol{N} \cdot\left(\boldsymbol{X}_{l}-\boldsymbol{X}\right)+\gamma_{1} \boldsymbol{N} \cdot\left(\boldsymbol{Y}_{l}-\boldsymbol{Y}\right)+\sigma_{p}^{\prime} L^{\kappa} .
$$

To illustrate model performance, consider the uniaxial cyclic behavior. Figure $7 \mathrm{a}, \mathrm{b}$ presents the evolution of back stresses $X$ and $Y$ for the case of cyclic loading controlled by harmonically varying stress of specified mean values $\sigma_{\mathrm{m}}=100$ or $300 \mathrm{MPa}$ and amplitude $\sigma_{\mathrm{a}}=800 \mathrm{MPa}$. In the case when the stress varies as 
(a)

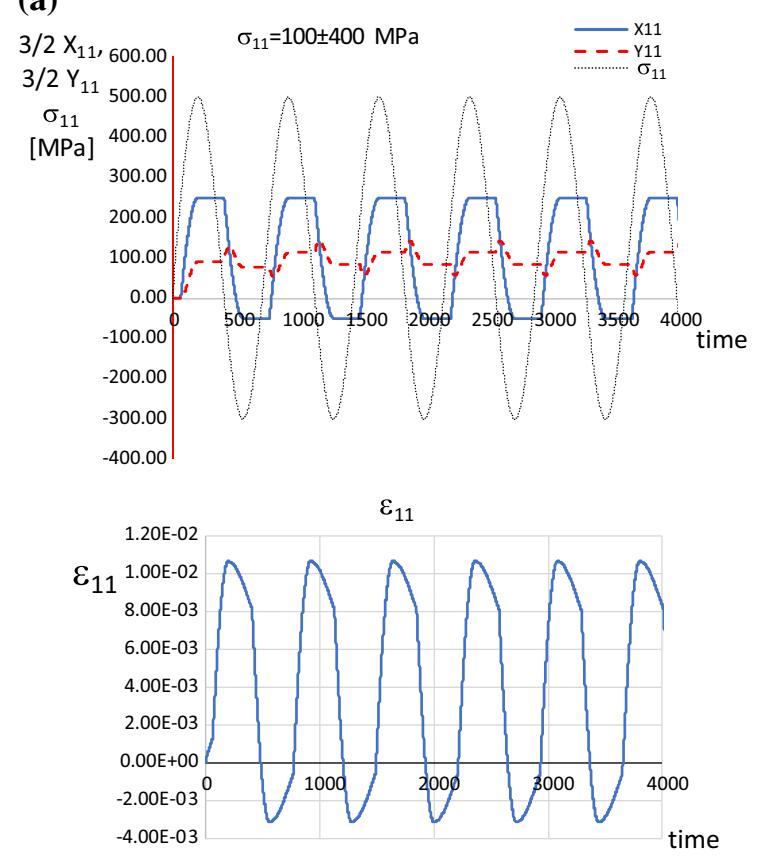

(b)
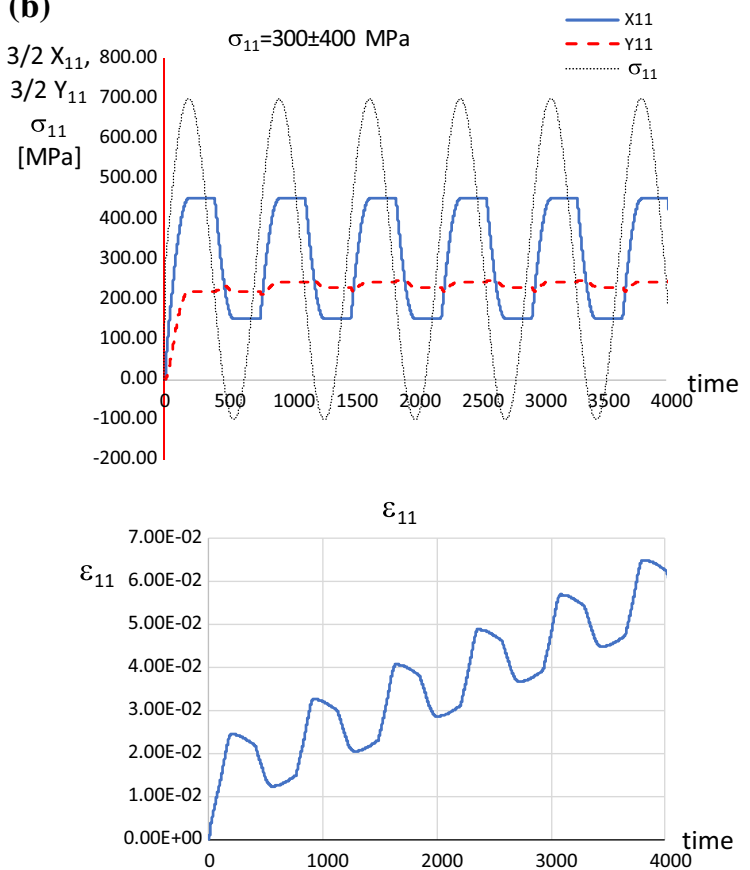

Fig. 7 Stress $\sigma_{11}$, backstress $\mathrm{X}_{11}, \mathrm{Y}_{11}$ and -strain $\varepsilon_{11}$ response in the cyclic tension-compression for the three-surface model; a $\sigma_{11}=100 \pm 400 \mathrm{MPa}, \mathbf{b} \sigma_{11}=300 \pm 400 \mathrm{MPa}$

$\sigma_{11}=100 \pm 400 \mathrm{MPa}$, there is no ratcheting effect, Fig. 7a. On the other hand, when the mean stress equals $300 \mathrm{Mpa}$, the ratcheting effects occurs in the steady state and the back stress $Y$ reaches its limit value $Y_{l}$, Fig. 7b. It was assumed that $\sigma_{p}=R_{l}=250 \mathrm{MPa}, \sigma_{l}=500 \mathrm{MPa}, \gamma=\gamma_{l}=100$.

It is noted that the present model formulation differs from the most frequently used assumption of expressing hardening effects in terms of additive set of back stresses satisfying AF evolution rule, cf. Chaboche review of cyclic plasticity models [19]. The present formulation is based on the concept of interacting evolution of back stress states and the coaxiality rule in steady-state regimes.

\section{Model application: simulation of uniaxial and biaxial cyclic response}

In this section, we shall apply the model to simulate cyclic response of some metals or alloys for uniaxial cyclic loading of specified strain amplitude and for the case of strain controlled axial extension of thin-walled tubes with superposed cyclic torsion of specified strain amplitude and rate of straining. The cyclic torsional straining is supposed to reduce the axial load and affect the grain structure. It was shown in the previous paper Mróz et al. [57] presenting the analysis for a perfectly plastic material that the axial stress reduction occurs at the expense of increased dissipated energy in the deformation process. However, for the hardening-recovery model discussed in this paper, the dissipated energy increase may be essentially reduced when the axial stress reduction is more significant.

To discuss the process parameters in the combined tension and cyclic torsion, consider a thin-walled tube of initial radius $r_{0}$, length $l_{0}$ and wall thickness $t_{0}$. Assume the axial strain rate to be specified and constant. The alternating torsion is imposed in order to reduce the axial stress and applied axial force required to execute the process. Denote by $\varepsilon_{x}$ and $\varepsilon_{x y}$ the axial and shear strain components and their rates by $\dot{\varepsilon}_{x}$ and $\dot{\gamma}_{x y}$. The Cauchy stress components are $\sigma_{x}, \tau_{x y}$. For simplicity, consider first the small strain formulation. The deformation program is shown in Fig. 8.

For uniform length variation, the axial strain and strain rate is $\varepsilon_{x}=\dot{\alpha} t, \dot{\varepsilon}_{x}=\dot{\alpha}$, where $\dot{\alpha}=i / l_{0}$. For the logarithmic strain measure there is $\varepsilon_{x}=\ln \left(l / l_{0}\right)=\ln (1+\dot{\alpha} t)$ and $\dot{\varepsilon}_{x}=i / l=\dot{\alpha} /(1+\dot{\alpha} t)$. The shear strain is assumed to oscillate within the range $2 \gamma_{m}$ and the period T. For piecewise linear oscillation, Fig. 8b, we have $\dot{\beta}=\dot{\gamma}_{x y}=4 \gamma_{m} / T$. Denote the ratio of rates of shear and axial strains by $\eta$, thus 

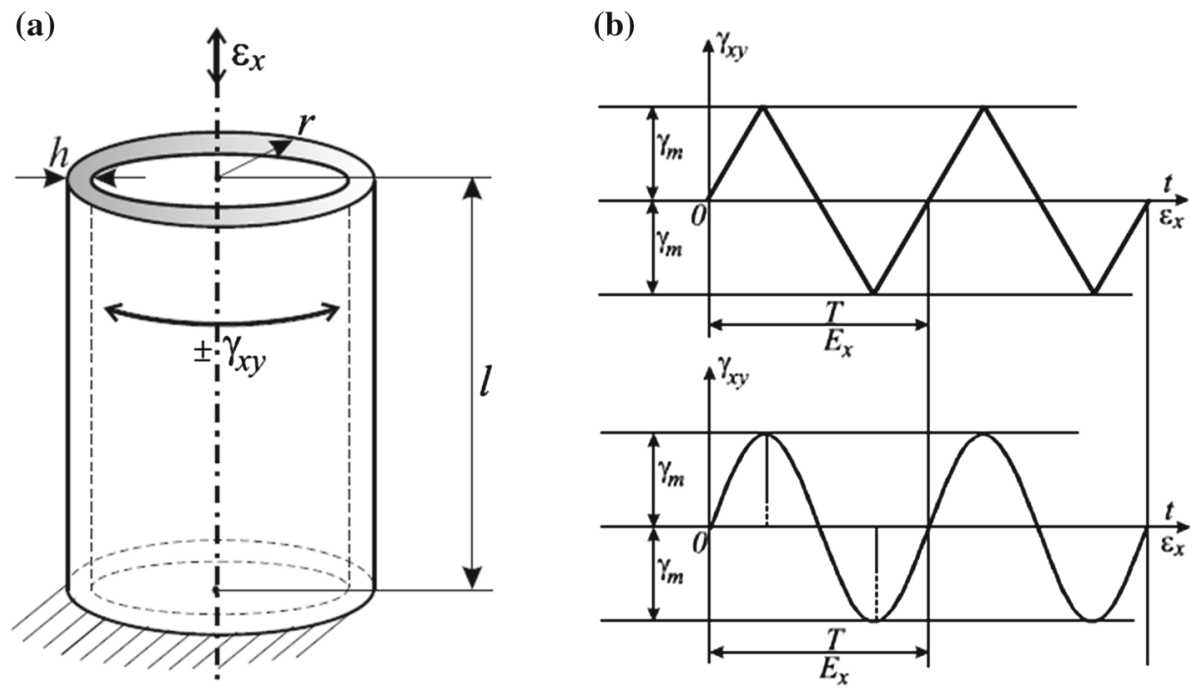

Fig. 8 Axial and torsional deformation program: a tube dimensions, b piecewise linear and harmonic variation of shear strain $\gamma_{x y}(\mathrm{t})$

$$
\eta=\frac{\dot{\beta}}{\dot{\alpha}}=\text { const }, \quad \dot{\beta}=\frac{4 \gamma_{m}}{T}, \dot{\beta}>0, \quad \dot{\alpha}>0
$$

For the harmonic variation of $\gamma_{x y}$, Fig. $10 \mathrm{~b}$, we can write

$$
\gamma_{x y}=\gamma_{m} \sin \left(\frac{2 \pi}{T} t\right), \quad \dot{\gamma}_{x y}=\frac{\pi}{2} \dot{\beta} \cos \left(\frac{2 \pi}{T} t\right), \quad \dot{\beta}=\frac{4 \gamma_{m}}{T},
$$

and the ratio of strain rates is

$$
\frac{\dot{\gamma}_{x y}(t)}{\left|\dot{\varepsilon}_{x}\right|}=\eta \frac{\pi}{2} \cos \left(\frac{2 \pi}{T} t\right)=\eta \frac{\pi}{2} \cos \left(\frac{2 \pi}{T} \frac{\left|\varepsilon_{x}\right|}{\dot{\alpha}}\right)=\eta \frac{\pi}{2} \cos \left(2 \pi \frac{\left|\varepsilon_{x}\right|}{E_{x}}\right),
$$

where $E_{x}=4 \gamma_{m} / \eta$ is the accumulated strain corresponding to the time period $T$. Let us note that the time measure can be replaced by the axial strain $\varepsilon_{x}=|\dot{\alpha}| t$.

For the piecewise linear and harmonic shear strain control, there are two essential parameters controlling the process, namely the strain rate ratio $\eta$ and the torsion amplitude $2 \gamma_{m}$. We shall study the cyclic deformation process for different values of $\eta$ and $\gamma_{m}$ after initial model calibration and verification for uniaxial cyclic loading.

The yield condition and the flow rule now are

$$
\begin{aligned}
& f_{p}=\sqrt{\left(\sigma_{x}-X_{x}\right)^{2}+3\left(\tau_{x y}-X_{x y}\right)^{2}}-\sigma_{p}=0, \\
& \dot{\varepsilon}_{x}^{p}=\dot{\lambda} \frac{\sigma_{x}-X_{x}}{\sigma_{p}}, \quad \dot{\gamma}_{x y}^{p}=\dot{\lambda} \frac{3\left(\tau_{x y}-X_{x y}\right)}{\sigma_{p}},
\end{aligned}
$$

where

$$
\dot{\lambda}=\sqrt{\left(\dot{\varepsilon}_{x}^{p}\right)^{2}+\frac{1}{3}\left(\dot{\gamma}_{x y}^{p}\right)^{2}}
$$

The constitutive rate equation for an elastic-plastic material have the form

$$
\begin{gathered}
\dot{\varepsilon}_{x}=\dot{\varepsilon}_{x}^{e}+\dot{\varepsilon}_{x}^{p}=\frac{\sigma_{x}}{E}+\dot{\lambda} \frac{\sigma_{x}-X_{x}}{\sigma_{p}}, \\
\dot{\gamma}_{x y}=\dot{\gamma}_{x y}^{e}+\dot{\gamma}_{x y}^{p}=\frac{\tau_{x y}}{G}+\dot{\lambda} \frac{3\left(\tau_{x y}-X_{x y}\right)}{\sigma_{p}}, \quad \dot{\lambda}>0, f_{p} \leq 0, \quad \dot{\lambda} f_{p}=0 .
\end{gathered}
$$


Table 1 Material parameters

\begin{tabular}{|c|c|c|c|c|c|c|c|c|c|c|c|c|c|c|}
\hline & $E(\mathrm{GPa})$ & $v(-)$ & $\sigma_{l 0}(\mathrm{MPa})$ & $\sigma_{l f}(\mathrm{MPa})$ & $s(-)$ & $w(-)$ & $k_{p}(-)$ & $\kappa(-)$ & $\gamma(-)$ & $k_{l}(-)$ & $\gamma_{l}(-)$ & $\beta(-)$ & $f(-)$ & $l_{0}(-)$ \\
\hline $\begin{array}{l}\text { Pure copper, } \\
\text { two-surface } \\
\text { model }\end{array}$ & 108 & 0.3 & 120 & 435 & 1 & 0.33 & 2.22 & 5 & 125 & - & - & 3 & - & 0 \\
\hline $\begin{array}{l}\text { Pure copper, } \\
\text { three-surface } \\
\text { model }\end{array}$ & 108 & 0.3 & 120 & 300 & 1 & 0.33 & 1.54 & 5 & 120 & 2.22 & 5.0 & 3 & 1.0 & 0 \\
\hline $\begin{array}{c}\text { Austenitic steel, } \\
\text { 0H18N9 }\end{array}$ & 150 & 0.3 & 420 & 830 & 1 & 0.3 & 1.54 & 6 & 60 & 5.0 & 5.0 & 3 & 1.0 & 0 \\
\hline $\begin{array}{l}\text { Aluminum alloy } \\
\text { PA7 }\end{array}$ & 77 & 0.4 & 480 & 540 & 1 & 10 & 1.25 & 0 & 705 & 2.87 & 45.0 & 6.7 & 1.0 & 0.1 \\
\hline
\end{tabular}

For the two-surface model, the hardening surfaces in the stress and back stress spaces are

$$
\begin{aligned}
& F_{l}(\sigma)=\sqrt{\sigma_{x}^{2}+3 \tau_{x y}^{2}}-\sigma_{l}(\xi)=0, \\
& F_{l}(X)=\sqrt{X_{x}^{2}+3 X_{x y}^{2}}-r_{l}(\xi)=0,
\end{aligned}
$$

and the limit saturation states are

$$
\begin{aligned}
& X_{x}^{l}=\frac{\sigma_{x}-X_{x}}{\sigma_{p}} r_{l}, \quad X_{x y}^{l}=\frac{\tau_{x y}-X_{x y}}{\sigma_{p}} r_{l}, \\
& \sigma_{x}^{l}=\left(\sigma_{x}-X_{x}\right)\left(1+\frac{r_{l}}{\sigma_{p}}\right), \quad \tau_{x y}^{l}=\left(\tau_{x y}-X_{x y}\right)\left(1+\frac{r_{l}}{\sigma_{p}}\right),
\end{aligned}
$$

so that Eq. (56) are satisfied. Similarly, for the three-surface model, the hardening and limit surfaces are

$$
\begin{aligned}
& F_{h}=\sqrt{\left(X_{x}-Y_{x}\right)^{2}+3\left(X_{x y}-Y_{x y}\right)^{2}}-\sigma_{l}(\xi)=0, \\
& F_{y}=\sqrt{Y_{x}^{2}+3 Y_{x y}^{2}}-R_{l}(\xi)=0,
\end{aligned}
$$

and the evolution rules for the back stresses $\boldsymbol{X}$ and $\boldsymbol{Y}$ are

$$
\begin{aligned}
& \dot{Y}_{x}=\dot{\lambda} \gamma_{1}\left(Y_{x}^{l}-Y_{x}\right), \quad \dot{X}_{x}=\dot{\lambda} \gamma\left(\sigma_{x}^{l}-\sigma_{x}\right)=\dot{\lambda} \gamma\left(X_{x}^{l}-X_{x}\right), \\
& \dot{Y}_{x y}=\dot{\lambda} \gamma_{1}\left(Y_{x y}^{l}-Y_{x y}\right), \quad \dot{X}_{x y}=\dot{\lambda} \gamma\left(\tau_{x y}^{l}-\tau_{x y}\right)=\dot{\lambda} \gamma\left(X_{x y}^{l}-X_{x y}\right) .
\end{aligned}
$$

The present model formulation can now be applied to simulate cyclic response of several materials.

\subsection{Uniaxial and biaxial cyclic response of copper}

For pure annealed copper, the experimental cyclic stress strain curves were obtained by Pawlicki and Grosman [65] in their experiments of thin-walled tubular specimens subjected to pure cyclic torsion and progressive tensile strain assisted by cyclic torsion. The model parameters for two-surface and three-surface models were calibrated obtained using hardening rule (26) and are presented in Table 1.

The cyclic torsion tests were carried out for three values of shear strain amplitudes $\Delta_{x y}=$ $0.032,0.06,0.144$. The experimental evolution of cyclic hardening toward steady states is shown in Fig. 9a; the representative model simulation is shown in Fig. 9b. It is seen that the model simulates fairly accurately the cyclic response of copper.

The application of model to the case of monotonic axial tension assisted by cyclic torsion is illustrated in Figs. 10 and 11 by applying two-surface and three-surface models.

Figure $10 \mathrm{a}, \mathrm{b}$ present the prediction of the axial stress variation by the two-surface hardening model for different values of rate parameter $\eta$ and shear strain amplitude $\gamma_{m}$. Increasing the parameter $\eta$ by increasing the frequency of torsional cycles with the constant rate of axial straining, the axial stress is reduced more 

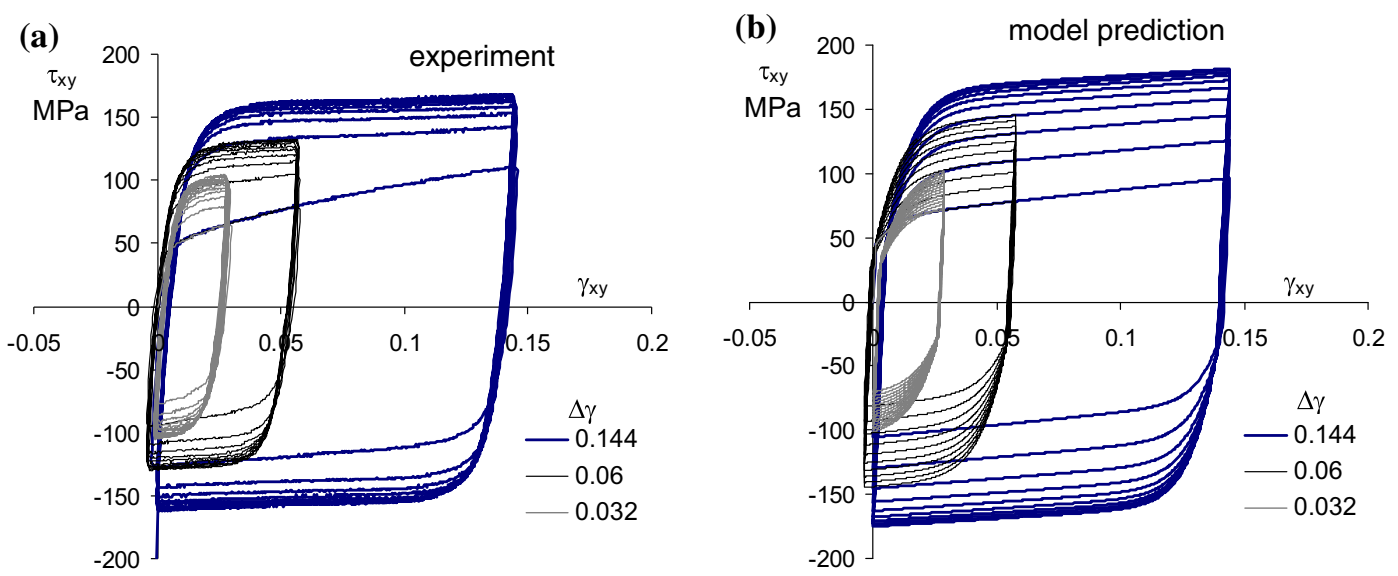

Fig. 9 Cyclic torsion response for three values of shear amplitudes $\Delta \gamma_{x y}=0.032,0.06,0.144$, a experimental data, b threesurface model prediction

(a)

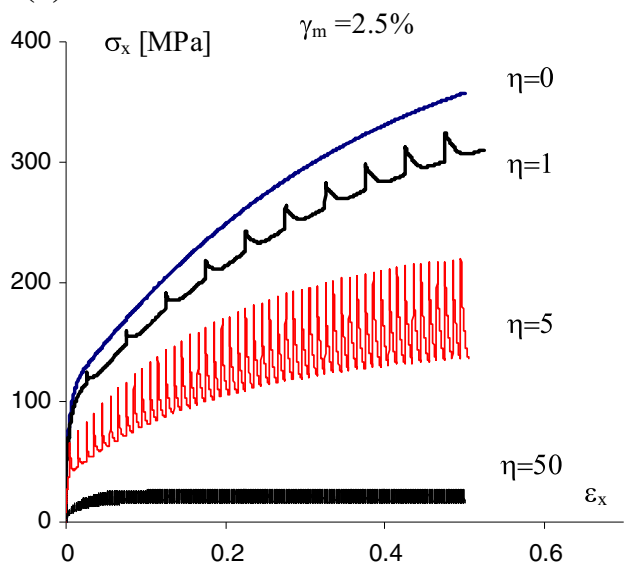

(b)

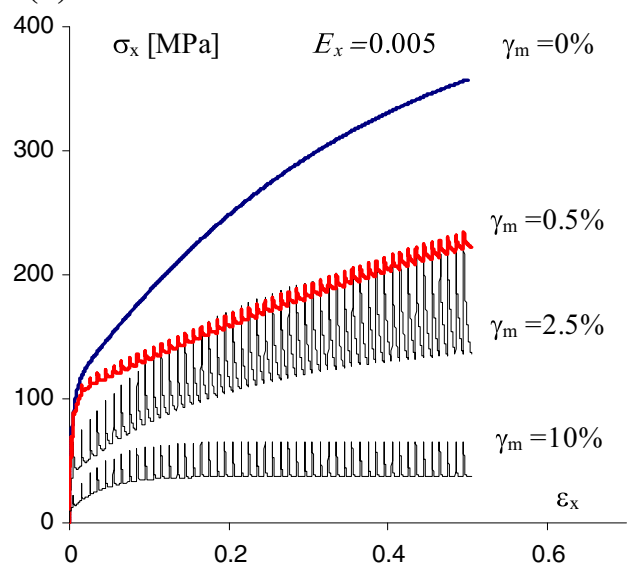

Fig. 10 Comparison of the tensile stress paths for piecewise linear shear strain control for different values $\eta$ and $\gamma_{m}$ for pure copper;-prediction by two-surface model: a prediction for $\gamma_{m}=2.5 \%$ and different values of $\eta$, b prediction for $E_{x}=0.005$ and different values of $\gamma_{m}$
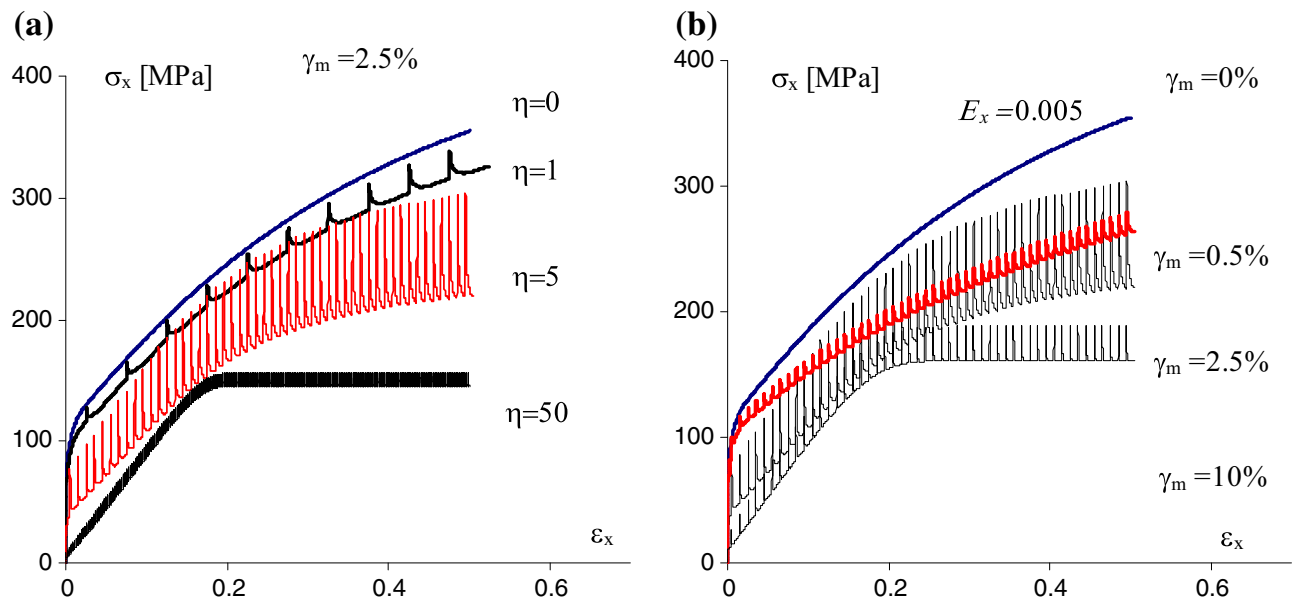

Fig. 11 Comparison of the tensile stress paths for piecewise linear shear strain control for different values $\eta$ and $\gamma_{m}$ for pure copper;- prediction by three-surface model: a prediction for $\gamma_{m}=2.5 \%$ and different values of $\eta$, $\mathbf{b}$ prediction for $E_{x}=0.005$ and different values of $\gamma_{m}$ 

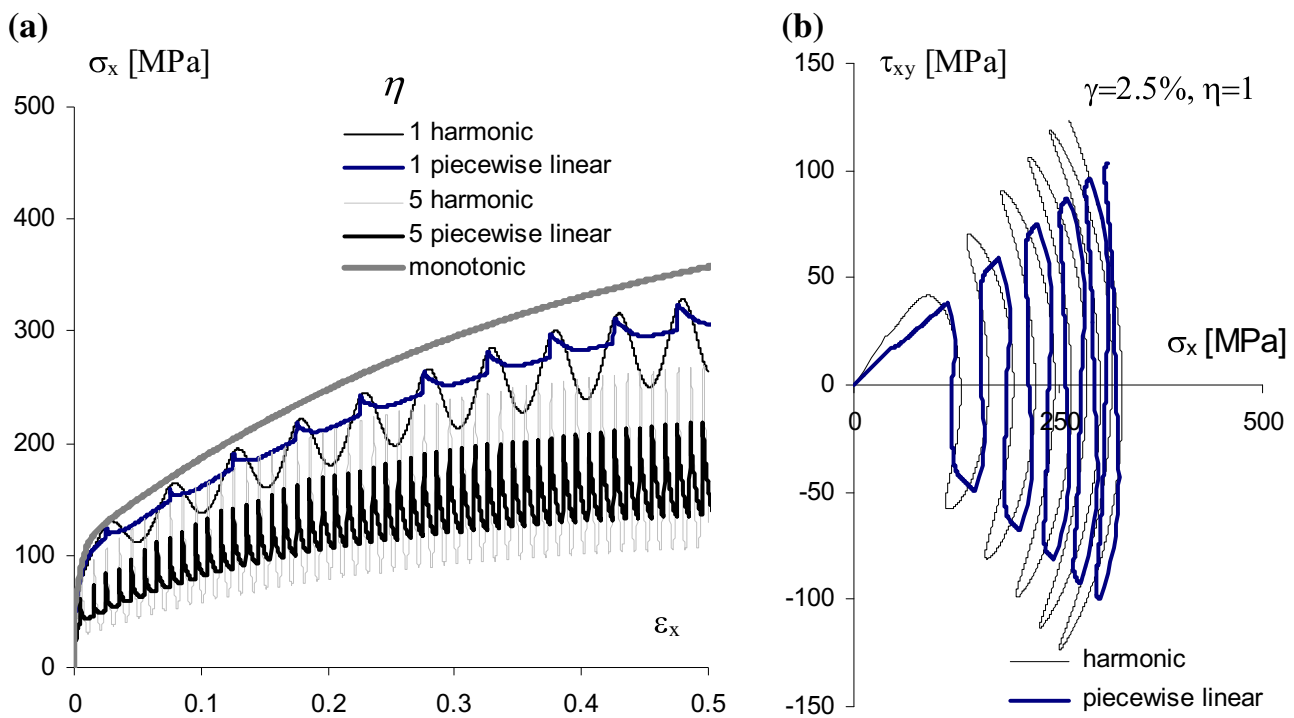

Fig. 12 Axial response for piecewise-linear and harmonic shear strain control for $\eta=0,1,5$ and $\gamma_{m}=0.025$; a axial stress evolution, $\mathbf{b}$ stress paths in the $\sigma_{\mathrm{x}}-\tau_{x y}$ plane

(a)

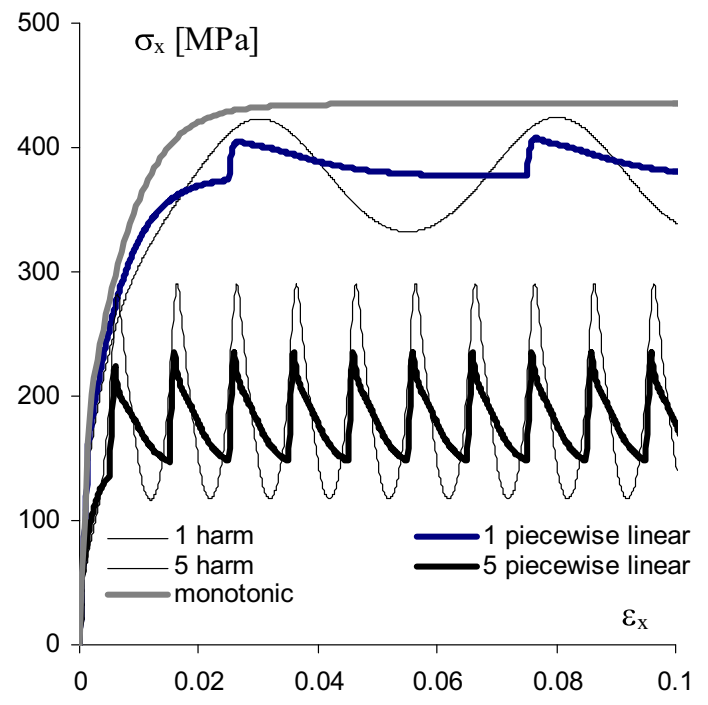

(b)

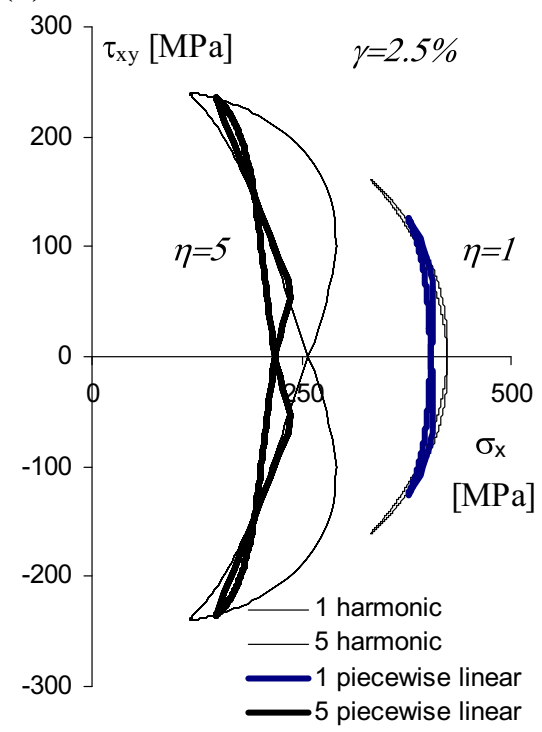

Fig. 13 Axial extension assisted by cyclic torsion for $\eta=0,1,5, \gamma_{m}=0.025$ and saturated isotropic hardening; a axial stress evolution, b stress paths in the $\sigma_{x}-\tau_{x y}$ plane

significantly for the higher values of $\eta$. However, for the two-surface model, the predicted axial stress reduction is much bigger than that predicted by three-surface model. In fact, for high values of $\eta$, the excessive reduction of $\sigma_{x}$ is predicted by the two surface model. Similarly, for increasing $\gamma_{m}$, the axial stress reduction is very large. However, the application of the three-surface model provides more accurate predictions. Figure 11 illustrates the axial stress variation for different values of $\eta$ and $\gamma_{m}$. The axial stress reduction for the same values of $\eta$ and $\gamma_{m}$ is much lower than that predicted by the two-surface model.

Figure 12 presents the predicted stress evolution for piecewise-linear and harmonic shear strain control for $\eta=0,1,5$ and $\gamma_{m}=0.025$. It is seen that the harmonic control is associated with higher stress amplitudes, Fig. 12a. The stress path evolution for both controls is shown in Fig. 12b. The two-surface model is applied assuming the evolution rule (26) and model parameters presented in Table 1. 
(a)

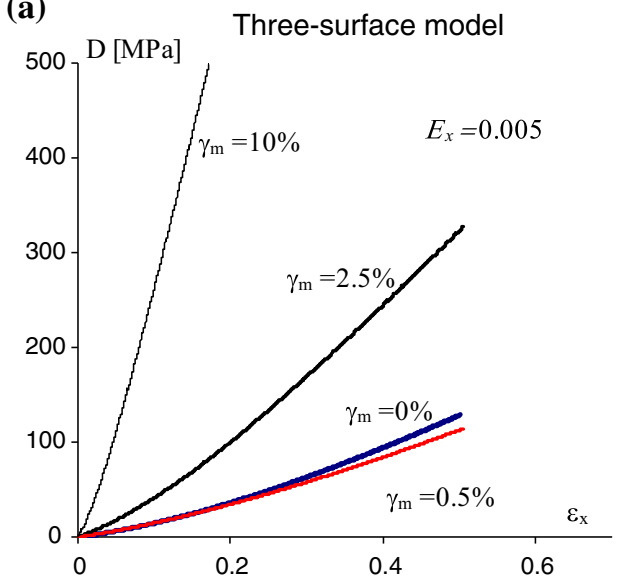

(b)

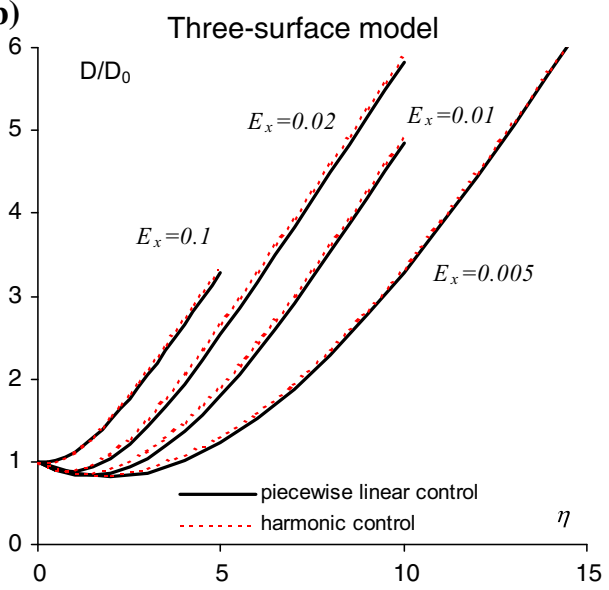

(c)

Three-surface model

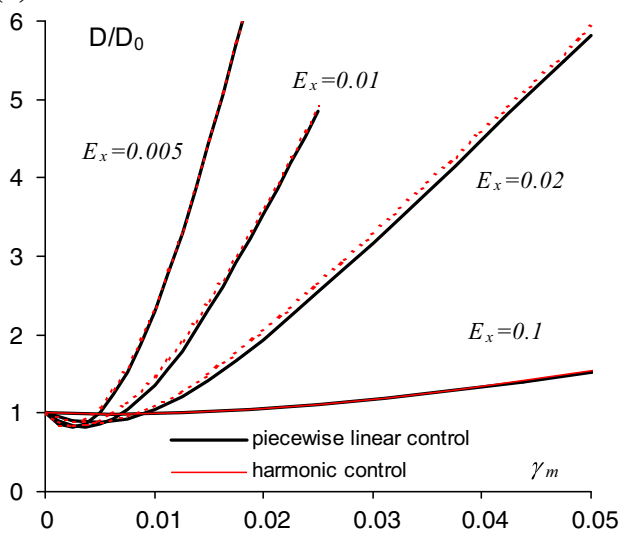

Fig. 14 Variation of the total plastic dissipation $D$ predicted by the three-surface model: a axial strain dependence for $E_{x}=0.005, \gamma_{m}=0,0.5,2.5,10 \%$ (or $\eta=0,4,2080$ ); $\mathbf{b}$ dependence of the ratio $D / D_{0}$ on the strain rate ratio $\eta$ for $E_{x}=0.005,0.01,0.02,0.1$; $\mathbf{c}$ dependence of the ratio $D / D_{0}$ on the shear strain amplitude $\gamma_{m}$ for $E_{x}=0.005,0.01,0.02,0.1$

A more clear comparison of predictions of stress evolution is shown in Fig. 13, where the hardening surface reaches its limit stress at small strain value and the material response is governed by kinematic hardening. In the isotropic hardening evolution rule (22), it is assumed that $\sigma_{l 0}=\sigma_{\max }=435 \mathrm{MPa}$. The stress path reaches the steady state with constant mean value of axial stress, Fig. 13a. The stress paths in the $\sigma_{x}-\sigma_{x y}$ plane are similar to those predicted for the perfectly plastic model, cf. Mróz et al. [57], with consecutive plastic and elastic states.

The total plastic dissipation of the process of axial extension assisted by cyclic torsion can be calculated and compared with the dissipation $D_{0}$ for the process of pure extension. Using the formula

$$
\begin{aligned}
D & =\int \sigma \cdot d \varepsilon^{p}=\int\left(\sigma_{x} d \varepsilon_{x}^{p}+\tau_{x y} d \gamma_{x y}^{p}\right), \\
D_{0} & =\int \sigma_{x} d \varepsilon_{x}^{p},
\end{aligned}
$$

the dissipated energy is calculated for both cyclic and monotonic deformation processes. Figure 14a presents the variation of $D$ with the axial strain for the strain amplitude $E_{x}=0.5 \%$ and different values of shear strain amplitude $\gamma_{m}$. It is seen that for $\gamma_{m}=0.5 \%$ (or $\eta=4$ ) the dissipated energy is lower than that for uniaxial extension. The variation of the ratio $D / D_{0}$ with $\eta$ and $\gamma_{m}$ is plotted in Fig. 14b, c. The effect of type of control of shear strain (harmonic and piecewise linear) on the energy dissipation in insignificant. These results differ essentially from the predictions of perfectly plastic model for which the energy dissipated in the cyclic deformation assisted by cyclic torsion was always greater than that for the proportional deformation process. 
This reduced energy dissipation is associated with the recovery effect lowering the stress level associated with plastic deformation.

Figure 15 presents the experimental and predicted shear stress evolutions for pure copper. The three-surface model prediction of stress-strain paths are compared with the experimental data of Pawlicki and Grosman [65]. The piecewise linear shear strain control was assumed in modeling; however, the actual strain control differed from piecewise linear and the axial stress evolution is not simulated accurately, though the mean stress evolution is well predicted.

\subsection{Modeling of cyclic response of aluminum alloy 2024}

The experimental program was executed by Kowalewski and Szymczak [50]. Their data are used for model calibration and prediction of cyclic response for combined tension and torsion. The commercial aluminum alloy 2024 used in aircraft industry exhibits initially the texture anisotropy affecting both elastic stiffness moduli and yield condition. The initial yield condition for the case tension and torsion is assumed in the form accounting for in-plane anisotropy, thus

$$
f_{p}=\sqrt{\sigma_{x}^{2}+\beta \tau_{x}^{2}}-\sigma_{p}=0
$$
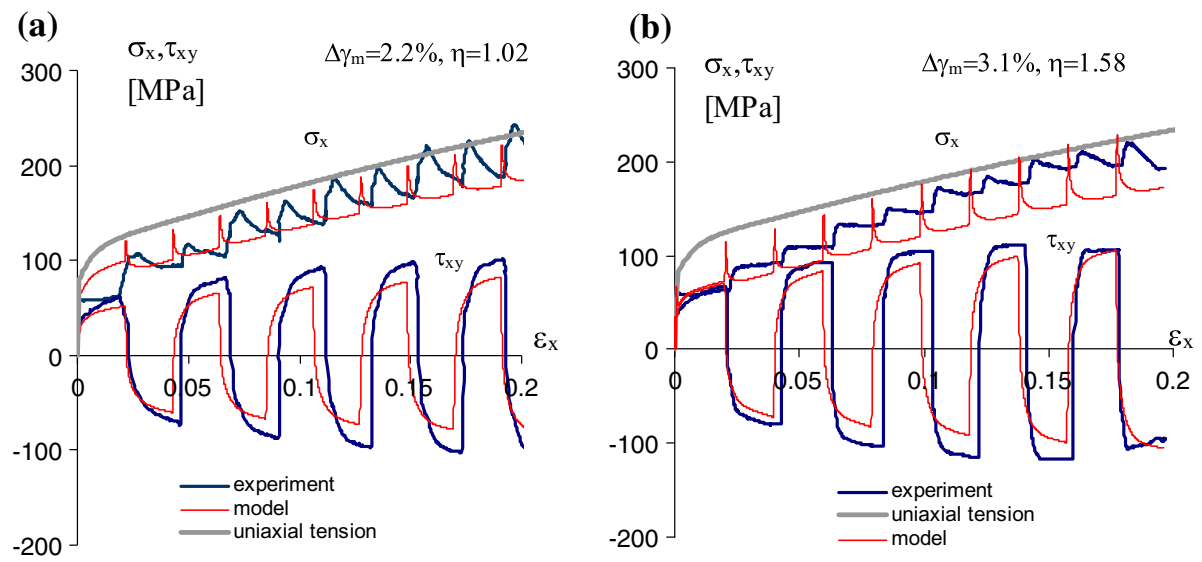

Fig. 15 Comparison of the three-surface model prediction with experimental data for axial extension assisted by cyclic torsion of pure copper for various $\eta$ : $\mathbf{a}$ axial and shear stress evolution for $\eta=1.02, \gamma_{m}=2.2 \%, \mathbf{b}$ axial and shear stress evolution for $\eta=1.58, \gamma_{m}=3.1 \%$

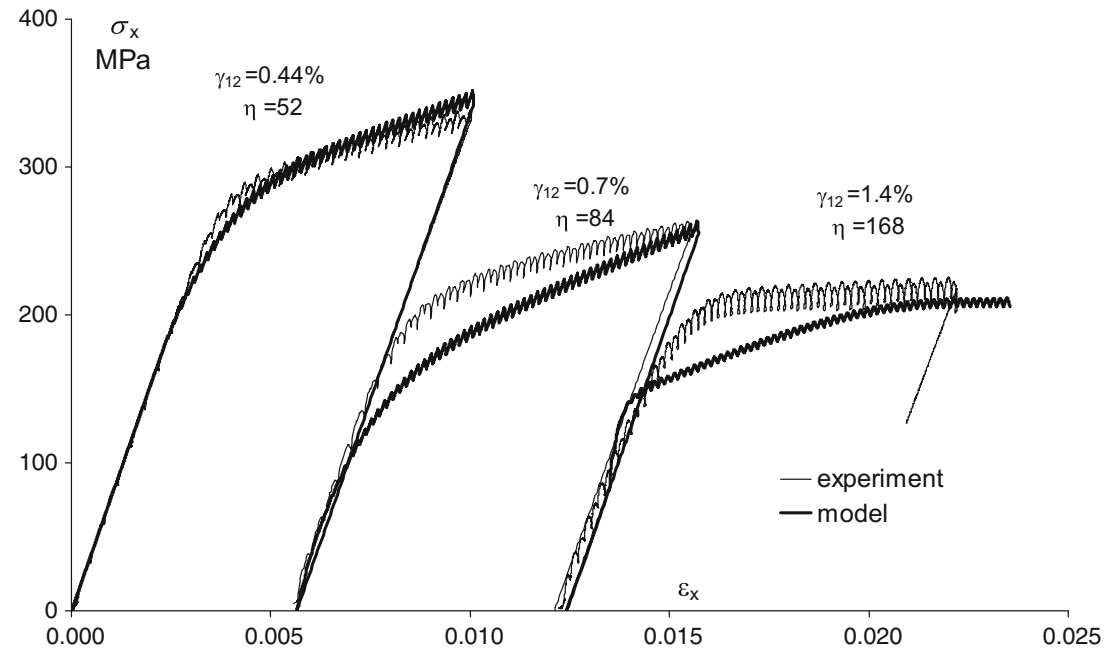

Fig. 16 Comparison of the three-surface model prediction with experimental data for axial extension with cyclic torsion of 2024 aluminum alloy for various process parameters: $\gamma_{\mathrm{m}}=0.44 \%, \eta=52 ; \gamma_{m}=0.7 \%, \eta=84$ and $\gamma_{m}=1.4 \%, \eta=168$ 
where the parameter $\beta=6.8$ instead of $\beta=3$ for the Huber-Mises condition. The elasticity moduli are $E=77 \mathrm{GPa}, \mathrm{G}=27 \mathrm{GPa}, v=0.32$. The model parameters for the two-surface and three-surface models have been calibrated from cyclic tests in tension and torsion. They are listed in Table 1.

The application of three-surface (3-S) model with material parameters calibrated from uniaxial cyclic tests to simulation of tension test assisted by cyclic torsion is demonstrated in Fig. 16. The same simulation was conducted by applying the two-surface model. The 3-S model provides much better simulation of axial stress evolution for the same values of $\gamma_{m}$ and $\eta$. The excessive axial stress reduction predicted by the two-surface model for large values of $\eta$ now is suppressed by assuming translation of the hardening surface center $\boldsymbol{Y}$ toward its limit position $\boldsymbol{Y}_{l}$.

\subsection{Modeling of cyclic response of austenitic steel (0H18N9)}

The material parameters of the three-surface model for the austenitic steel were calibrated from the torsion cyclic test. The model parameters for three-surface models are listed in Table 1.

Figure 17 presents the experimental and predicted cyclic stress-strain curves for three values of strain amplitude. The predicted axial stress evolution in the extension test assisted by cyclic torsion is shown in
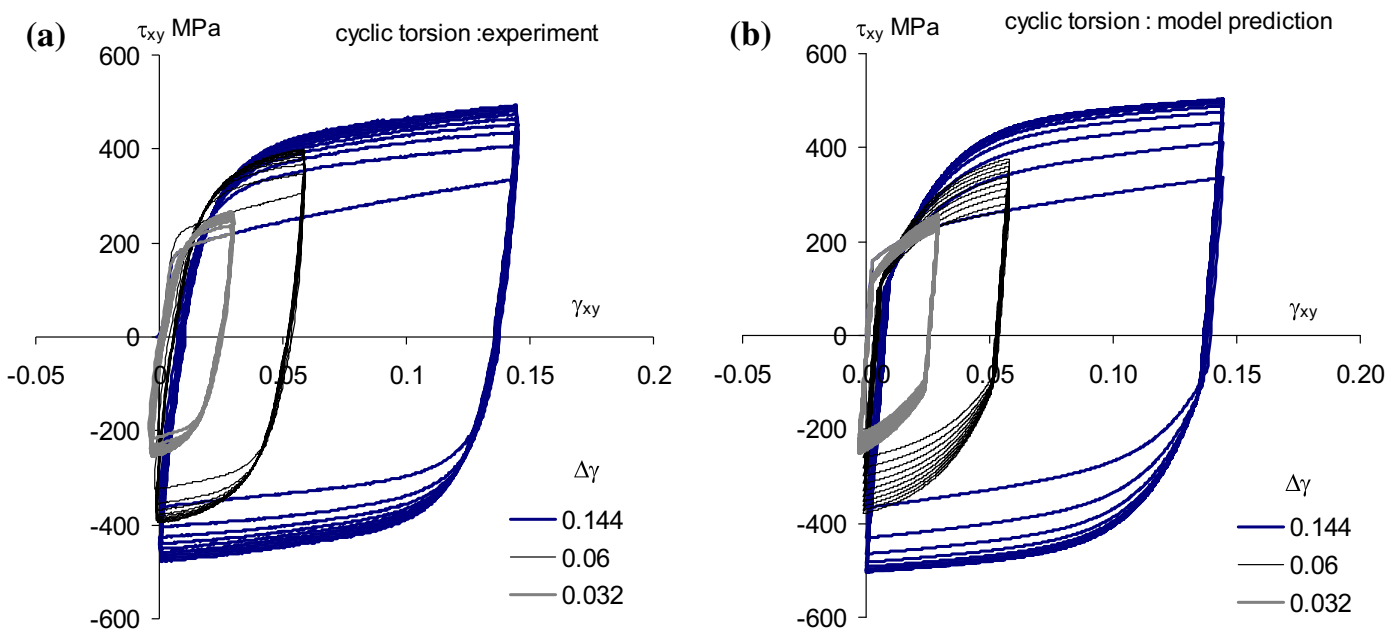

Fig. 17 Austenitic steel: cyclic torsion response for three values of shear amplitudes $\Delta \gamma_{x y}=0.032,0.06,0.144$, a experimental data after Pawlicki and Grosman [65], b model prediction

(a)

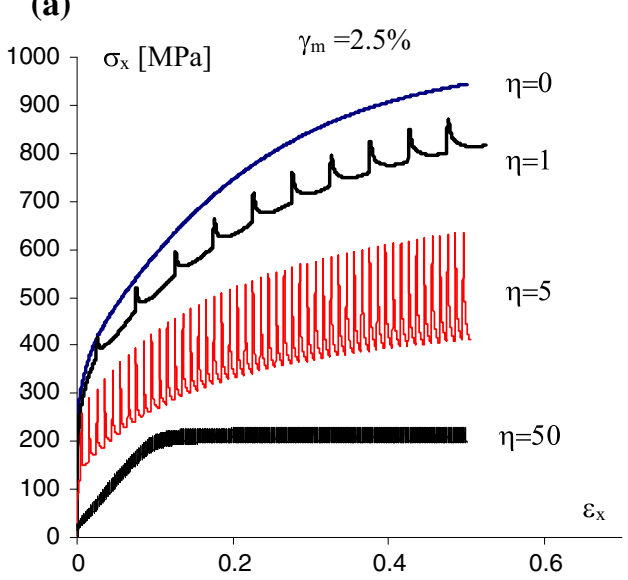

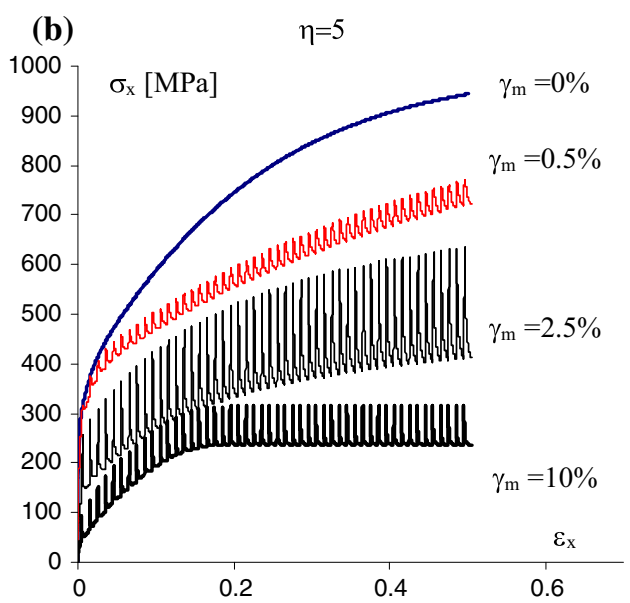

Fig. 18 Austenitic steel: The predicted axial extension assisted by cyclic torsion for several values of $\gamma_{m}$ and $\eta$ : a constant shear strain amplitude $\gamma_{\mathrm{m}}=2.5 \%$, varying $\eta=0,1,5,50 ; \mathbf{b}$ constant strain rate ratio $\eta=5$, varying shear strain amplitude $\gamma_{m}=0 \%, 0.5,2.5,10 \%$ 
Fig. 18 for several values of $\gamma_{m}$ and $\eta$. The experimental verification of this prediction is at present not available.

Recently, extensive cyclic loading tests for strain controlled non-proportional deformation programs were conducted by Kowalewski et al. [49,51] for pure copper, aluminum alloy and high strength steel. In particular, for circular and square strain paths, additional hardening and stress retardation effects were observed. The present 3-S constitutive model was applied to simulate the deformational response with satisfactory results.

\section{Concluding remarks}

In most papers related to cyclic plasticity, the material response for stress or mixed controlled processes was analyzed for both proportional and non-proportional loading. The analysis of transient and steady cyclic states for ratcheting and shake-down responses constituted the main objective of research directed to application in structural design. The low cycle fatigue and excessive accumulation of plastic strain may then generate essential failure modes to be considered in safe design. On the other hand, in technological processes, the cyclic deformation and ratcheting effect can be beneficial in assistance of the main deformation process by reducing the load required for its operation. The present paper is aimed at the formulation of a simple model able to simulate the cyclic loading response for the case of progressive plastic deformation assisted by cyclic straining. The strain controlled cyclic deformation process is then considered. The model is aimed to be applied in numerical analysis of such processes as extrusion, forging and rolling, assisted by cyclic shear deformation. It is seen that a considerable axial stress reduction is attained for the properly selected shear strain amplitude $\gamma_{m}$ and the strain ratio $\eta$. The two-surface model predicts too excessive reduction of axial stress, not confirmed experimentally. On the other hand, the three-surface model provides realistic predictions and can be applied to predict cyclic response of different materials. The interesting result is also obtained, namely the reduced plastic energy dissipation required to extend the element to a specified strain value for some range of parameters $\gamma_{m}$ and $\eta$, as compared to the monotonic deformation process. The microstructure, texture and grain size evolution during the cyclic deformation process was not discussed in the paper as these topics require further material research.

The present model is based on the concept of hardening and recovery expressed in terms of two back stresses interacting in their evolution and tending to coaxiality in their steady states. Such effects as cyclic strain amplitude or frequency-dependent hardening and non-proportional loading induced additional hardening can be effectively simulated by the 3-S model as has been demonstrated in [49]. The model can also be applied in simulation of ratcheting effects for stress or mixed controlled cyclic loading programs. This problem will be discussed in a separate paper. Referring to numerous constitutive models simulating cyclic ratcheting of metals, the present formulation seems simpler involving a limited numbers of material parameters, easy to identify from uniaxial or biaxial cyclic tests.

Open Access This article is distributed under the terms of the Creative Commons Attribution 4.0 International License (http:// creativecommons.org/licenses/by/4.0/), which permits unrestricted use, distribution, and reproduction in any medium, provided you give appropriate credit to the original author(s) and the source, provide a link to the Creative Commons license, and indicate if changes were made.

\section{References}

1. Abdel-Karim, M., Khan, A.: Cyclic multiaxial and shear finite deformation responses of OFHC Cu. Part II: an extension to the KHL model and simulations. Int. J. Plast. (2009). doi:10.1016/j.ijplas.2009.10.008

2. Abdel-Karim, M.: An evaluation for several kinematic hardening rules on prediction of multiaxial stress controlled ratcheting. Int. J. Plast. 26, 711-730 (2009)

3. Abdel-Karim, M.: Modified kinematic hardening rules for simulations of ratcheting. Int. J. Plast. 25, 1560-1587 (2009)

4. Abdel-Karim, M.: Shakedown of complex structures according to various hardening rules. Int. J. Press. Vessels Pip. 82, 427-458 (2005)

5. Abdel-Karim, M., Ohno, N.: Kinematic hardening model suitable for ratcheting with steady-state. Int. J. Plast. 16, 225-240 (2000)

6. Abdel-Karim, M.: Numerical integration method for kinematic hardening rules with partial activation of dynamic recovery term. Int. J. Plast. 21, 1303-1321 (2005)

7. Armstrong, P.J., Frederick, C.O.: A mathematical representation of the multiaxial Bauschinger effect, CEGB Report RD/B/N731, Berkeley Nuclear Laboratories (1966) 
8. Bari, S., Hassan, T.: An advancement in cyclic plasticity modeling for multiaxial ratcheting simulation. Int. J. Plast. 18, 873-894 (2002)

9. Bochniak, W., Korbel, A.: Extrusion of CuZn39Pb2 alloy by the KOBO method. Eng. Trans. 47, 351-367 (1999)

10. Bochniak, W., Korbel, A.: KOBO-type forming: forging of metals under complex conditions of the process. J. Mat. Process. Technnol. 134, 120-134 (2003)

11. Bochniak, W., Korbel, A.: Lüders deformation and superplastic flow of metals extruded by KOBO method. Philos. Mag. 93, 1883-1913 (2013)

12. Bochniak, W., Korbel, A.: Plastic flow of aluminum extruded under complex conditions. Mater. Sci. Technol. 16, 664-674 (2000)

13. Bouvier, S., Alves, J.L., Oliveira, M.C., Menezes, L.F.: Modelling of anisotropic work-hardening behaviour of metallic materials subjected to strain path changes. Comput. Mater. Sci. 32, 301-315 (2005)

14. Burlet, H., Cailletaud, G.: Numerical techniques for cyclic plasticity at variable temperature. Eng. Comput. 3, 143-153 (1986)

15. Chaboche, J.L., Kanoute, P., Azzouz, F.: Cyclic inelastic constitutive equations and their impact on the fatigue life predictions. Int. J. Plast. 35, 44-66 (2012)

16. Chaboche, J.L., Nouailhas, D.: A unified constitutive model for cyclic viscoplasticity and its application to various stainless steels. ASME J. Eng. Mater. Technol. 111, 424-430 (1989)

17. Chaboche, J.L., Nouailhas, D.: Constitutive modeling of ratcheting effects: I. Experimental facts and properties of the classical models, II. possibilities of some additional kinematic rules. ASME. J. Eng. Mater. Technol. 111, 384-416 (1989)

18. Chaboche, J.L., Rousselier, R.G.: On the plastic and viscoplastic constitutive equations, Part I : rules developed with internal variable concepts, Part II: application of internal variable concepts to the 316 stainless steel. ASME. J. Press. Vessel Technol. 105, 153-158, 159-164 (1983)

19. Chaboche, J.L.: A review of some plasticity and viscoplasticity constitutive theories. Int. J. Plast. 24, 1642-1693 (2008)

20. Chaboche, J.L.: Modeling of ratcheting: evaluation of various approaches. Eur. J. Mech. A Solids 13, 501-518 (1994)

21. Chaboche, J.L.: On some modifications of kinematic hardening to improve the description of ratcheting effects. Int. J. Plast. 7, 661-678 (1991)

22. Chaboche, J.L.: Time-independent constitutive theories for cyclic plasticity. Int. J. Plast. 2, 149-188 (1986)

23. Chen, X., Jiao, R., Kim, K.S.: On the Ohno-Wang kinematic hardening rules for multiaxial ratcheting modeling of medium carbon steel. Int. J. Plast. 21, 161-184 (2005)

24. Chen, X., Jiao, R., Kim, K.S.: Simulation of ratcheting strain to a high number of cycles under multiaxial loading. Int. J. Solids Struct. 40, 7449-7461 (2003)

25. Choi, Yangwook, Han, Chung-Souk, Lee, June K., Wagoner, Robert H.: Modeling multi-axial deformation of planar anisotropic elasto-plastic materials, part I: theory. Int. J. Plast. 22, 1745-1764 (2006)

26. Chu, C.C.: A three-dimensional model of anisotropic hardening in metals and its application to the analysis of sheet metal formability. J. Mech. Phys. Solids 32, 197-212 (1984)

27. Chu, C.C.: The analysis of multiaxial cyclic problems with an anisotropic hardening model. Int. J. Solids Struct. 23, 569-579 (1987)

28. Dafalias, Y.F., Kourousis, K.I., Saridis, G.J.: Multiplicative AF kinematic hardening in plasticity. Int. J.Solids Struct. 45, 2861-2880 (2008)

29. Dafalias, Y.F., Popov, E.P.: Plastic internal variable formalism of plasticity. ASME J. Appl. Mech. 98, 645-651 (1976)

30. Feaugas, X., Gaudin, C.: Ratcheting process in the stainless steel AISI 316L at $300 \mathrm{~K}$ : an experimental investigation. Int. J. Plast. 21, 643-662 (2004)

31. Feigenbaum, H.P., Dafalias, Y.F.: Biaxial ratcheting with novel variations of kinematic hardening. Int. J. Plast. 27, 479-491 (2010)

32. Follansbee, P.S., Kocks, U.F.: A constitutive description of the deformation of copper based on the use of the mechanical threshold stress as an internal state variable. Acta Metall. 36, 81-93 (1988)

33. Hassan, T., Taleb, L., Krishna, S.: Influence of non-proportional loading on ratcheting responses and simulations by two recent cyclic plasticity models. Int. J. Plast. 24, 1863-1889 (2009)

34. Hassan, T., Kyriakides, S.: Ratchetting of cyclically hardening and softening materials: I. Uniaxial behaviour, II. Multiaxial behaviour. Int. J. Plast. 10, 149-212 (1994)

35. Jiang, Y.Y., Sehitoglu, H.: Cyclic ratcheting of 1070 steel under multiaxial stress state. Int. J. Plast. 10, 579-608 (1994)

36. Kang, G., Liu, Y., Ding, J., Gao, Q.: Uniaxial ratcheting and fatigue failure of tempered 42CrMo steel: Damage evolution and damage-coupled visco-plastic constitutive model. Int. J. Plast. 25, 838-860 (2009)

37. Kang, G., Kan, Q.H., Zhang, J., Sun, Y.F.: Time-dependent ratchetting experiments of SS304 stainless steel. Int. J. Plast. 22, 858-894 (2006)

38. Kang, G., Ohno, N., Nebu, A.: Constitutive modeling of strain range dependent cyclic hardening. Int. J. Plast. 19, 1801-1819 (2003)

39. Kang, G.Z., Gao, Q., Yang, X.J.: A visco-plastic constitutive model incorporated with cyclic hardening for uniaxial/multiaxial ratcheting of SS304 stainless steel at room temperature. Mech. Mater. 34, 521-531 (2002)

40. Kang, G.Z., Gao, Q., Yang, X.J.: Uniaxial and multiaxial ratchetting of SS304 stainless steel at room temperature: experiments and visco-plastic constitutive model. Int. J. Nonlin. Mech. 39, 843-857 (2004)

41. Kang, G.Z.: Ratchetting: recent progresses in phenomenon observation, constitutive modeling and application. Int. J. Fatigue 30, 1448-1472 (2008)

42. Khan, A.S., Kazmi, R., Pandey, A., Stoughton, T.: Evolution of subsequent yield surfaces and elastic constants with finite plastic deformation. Part-I: a very low work hardening aluminum alloy (Al6061-T6511). Int. J. Plast. 25, 1611-1625 (2009)

43. Khan, A.S., Wang, X.: On non-proportional infinitesimal plastic deformation after finite plastic prestraining and partial unloading. J. Mech. Phys. Solids 36, 511-535 (1988)

44. Khoei, A.R., Jamali, N.: On the implementation of a multi-surface kinematic hardening plasticity and its applications. Int. J. Plast. 21, 1741-1770 (2005) 
45. Klisiński, M.: Plasticity theory based on fuzzy stress. ASME J. Eng. Mech. 114, 563-582 (1988)

46. Kong, L.X., Hodgson, P.D., Wang, B.: Development of constitutive models for metal forming with cyclic strain softening. J. Mater. Process. Technol 89-90, 44-50 (1999)

47. Korbel, A., Bochniak, W.: Refinement and control of metal structure elements by plastic deformation. Scr. Mater. 51, 755-759 (2004)

48. Kowalczyk-Gajewska, K., Mróz, Z., Pęcherski, R.: Micromechanical modeling of polycrystalline materials under nonproportional deformation paths. Arch. Metall. Mater. 52, 181-192 (2007)

49. Kowalewski, Z.L., Szymczak, T., Maciejewski, J.: Material effects during monotonic-cyclic loading. Int J. Solids Struct. 51, 740-753 (2014)

50. Kowalewski, Z.L., Szymczak, T.: On the mutual interactions of monotonic and cyclic loading and their effect on the strength of aluminum alloys. Appl. Mech. Mater. 2, 213-21 (2010)

51. Kowalewski, Z.L., Szymczak, T., Maciejewski, J.: Experimental and numerical investigations of the effects associated to complex loading combinations. In: Altenbach, H., Bruning, M. (eds.) Inelastic Behavior of Materials and Structures Under Monotonic and Cyclic Loading, pp. 117-142. Springer, Berlin (2015)

52. Krieg, R.D.: A practical two surface plasticity theory. ASME J. Appl. Mech. 42, 641-646 (1975)

53. Lemaitre, J., Chaboche, J.L.: Mechanics of Materials. Cambridge University Press, Cambridge (1989)

54. Maciejewski, J., Mróz, Z.: An upper-bound analysis of axisymmetric extrusion assisted by cyclic torsion. J. Mater. Process. Technol. 206, 333-344 (2008)

55. McDowell, D.L.: A two surface model for transient nonproportional cyclic plasticity. ASME J. Appl. Mech. 52, 298-308 (1985)

56. Moosbrugger, J.C., McDowell, D.L.: On a class of kinematic hardening rules for nonproportional cyclic plasticity. ASME. J. Eng. Mater. Technol. 111(1), 87-98 (1989)

57. Mróz, Z., Kowalczyk-Gajewska, K., Maciejewski, J., Pęcherski, R.: Tensile or compressive plastic deformation of cylinders assisted by cyclic torsion. Arch. Mech. 58, 497-527 (2006)

58. Mróz, Z.: On the description of anisotropic workhardening. J. Mech. Phys. Solids 15, 163-175 (1967)

59. Mróz, Z.: Phenomenological constitutive models for metals. In: Zarka, J., Gittus, J. (eds.) Modelling of Small Deformations of Metals, pp. 293-347. Elsevier, Amsterdam (1986)

60. Mróz, Z., Rodzik, P.: On multisurface and integral description of anisotropic hardening evolution of metals. Eur. J. Mech. A Solids 15, 1-28 (1996)

61. Ohno, N., Kachi, Y.: A constitutive model of cyclic plasticity for nonlinear hardening materials. ASME J. Appl. Mech. 53, 395-403 (1986)

62. Ohno, N.: Constitutive modeling of cyclic plasticity with emphasis on ratcheting. Int. J. Mech. Sci. 40, 251-261 (1998)

63. Ohno, N.: Recent topics in constitutive modeling of cyclic plasticity and viscoplasticity. Appl. Mech. Rev. 43, 283-295 (1990)

64. Ohno, N., Wang, J.D.: Transformation of a nonlinear kinematic hardening rule to a multisurface form under isothermal and nonisothermal conditions. Int. J. Plast. 7, 879-891 (1992)

65. Pawlicki, J., Grosman, F.: The impact of compression with oscillatory torsion parameters on technological plasticity of metals. Rudy Metale 50, 590-594 (2005)

66. Portier, L., Calloch, S., Marquis, D., Geyer, P.: Ratchetting under tension-torsion loadings: experiments and modeling. Int. J. Plast. 16, 303-335 (2000)

67. Press. Vessel Technol. 105, 153-164 (1983)

68. Sai, K., Cailletaud, G.: Multimechanism models for the description of ratchetting: effect of the scale transition rule and the coupling between hardening variables. Int. J. Plast. 23, 1589-1617 (2007)

69. Tanaka, E., Murakami, S., Ooka, M.: Effects of strain paths shapes on proportional cyclic plasticity. J. Mech. Phys. Solids 33, 559-575 (1985)

70. Trạmpczyński, W., Mróz, Z.: Anisotropic hardening model and its application to cyclic loading. Int. J. Plast. 8, 925-946 (1992)

71. Tseng, N.T., Lee, G.C.: Simple plasticity model of two-surface type. J. Eng. Mech. 109, 795-810 (1983)

72. Velay, D.L.: On class of kinematic hardening rules for non-proportional cyclic plasticity. J. Eng. Mater. Technol. 111, 87-98 (1987)

73. Velay, V., Bernhart, G., Penazzi, L.: Cyclic behavior modeling of a tempered martensitic hot work tool steel. Int. J. Plast. 22, 459-496 (2006)

74. Vincent, L., Calloch, S., Marquis, D.: A general cyclic plasticity model taking into account yield surface distortion for multiaxial ratcheting. Int. J. Plast. 20, 1817-1850 (2004)

75. Voyiadjis, G.Z., Basuroychowdhury, I.N.: A plasticity model for multiaxial cyclic loading and ratchetting. Acta Mech. 126, 19-35 (1998)

76. Yoshida, F., Uemori, T.: A model of large-strain cyclic plasticity describing the Bauschinger effect and workhardening stagnation. Int. J. Plast. 18, 661-686 (2002) 